\title{
Numerical modeling of undersea acoustics using a partition of unity method with plane waves enrichment
}

\author{
Raúl Hospital-Bravo • Josep Sarrate • Pedro Díez
}

Received: date / Accepted: date

\begin{abstract}
A new 2D numerical model to predict the underwater acoustic propagation is obtained by exploring the potential of the Partition of Unity Method (PUM) enriched with plane waves. The aim of the work is to obtain sound pressure level distributions when multiple operational noise sources are present, in order to assess the acoustic impact over the marine fauna. The model takes advantage of the suitability of the PUM for solving the Helmholtz equation, especially for the practical case of large domains and medium frequencies. The seawater acoustic absorption and the acoustic reflectance of the sea surface and sea bottom are explicitly considered, and Perfectly Matched Layers (PML) are placed at the lateral artificial boundaries to avoid spurious reflexions. The model includes semi-analytical integration rules which are adapted to highly oscillatory integrands with the aim of reducing the computational cost of the integration step. In addition, we develop a novel strategy to mitigate the ill-conditioning of the elemental and global system matrices. Specifically, we compute a low-rank approximation of the local space of solutions, which in turn reduces the number of degrees of freedom, the CPU time and the memory footprint. Numerical examples are presented to illustrate the capabilities of the model and to assess its accuracy.
\end{abstract}

Keywords Underwater acoustic propagation · Helmholtz equation · Partition of Unity Method ·

This work is partially supported by KIC InnoEnergy and European Institute of Innovation and Technology (EIT) through project Offshore Test Station (OTS; 03_2011_LH03 Industry Energy Efficiency).

Laboratori de Càlcul Numèric (LaCàN), Departament de Matemàtica Aplicada III, Universitat Politècnica de Catalunya, Jordi Girona 1, E-08034 Barcelona, Spain. E-mail: raul.hospital@upc.edu, jose.sarrate@upc.edu, pedro.diez@upc.edu
Plane waves - Complex wavenumber - Low-rank approximation · Singular value decomposition

\section{Introduction}

The acoustic pollution due to anthropogenic activities in the oceans, such as seismic exploration, military sonar operation, commercial shipping, construction, oil and gas extraction or offshore energy generation, has a direct impact on marine ecosystems. Until recent years, there was a lack of knowledge on the environmental impact of the underwater noise, and even nowadays special efforts are focused on monitoring the underwater noise levels $[1,2]$, identifying and characterizing the effects of the noise on the marine endangered species $[3$, 4], and establishing criteria to assess this impact [5]. For instance, a high level of exposure produces behavioral changes, masking of sounds of interest, hearing losses and temporary or permanent injuries over the marine fauna. Therefore, a growing social concern on this issue materialized in several national and international agreements and regulations that identify the anthropogenic noise as a specific type of pollution [6,7], and that plan to establish limits on the generated noise intensity [8]. In particular, Descriptor 11 from the European Union Commission Decision 2010/477/EU requires future monitoring programs to assess the underwater noise with frequencies ranging between $10 \mathrm{~Hz}$ and $10 \mathrm{kHz}$.

In this context, underwater acoustic propagation models gain importance as they provide a priori spatial distributions of the sound levels in the region of interest, which are crucial in the elaboration of acoustic impact assessments [9]. This work focuses on the simulation of the propagation of the noise produced by mul- 
tiple non-impulsive operational sources through large domains (from hundreds of meters to several kilometers) at medium frequencies (from hundreds of $\mathrm{Hz}$ to a few $\mathrm{kHz}$ ).

Several numerical methods have been developed to predict the sound pressure level under these conditions. For instance, range-dependent simplified methods [10] provide a first approximation by estimating the transmission loss due to the spreading and the seawater absorption. Methods based on a parabolic simplification of the Helmholtz equation produce better results even though they assume that energy propagates nearly horizontally, the speed of sound varies weakly and outgoing energy dominates backscattered energy $[11,12]$. However, full wave models give more realistic results since the wave physics is considered and no additional assumptions are made on the wave propagation behavior. When non-impulsive noise sources are addressed, full wave models consider the Helmholtz equation, which is the harmonic version of the wave equation. To this end, each input noise spectrum is decomposed into a set of harmonic frequency bands, and a single simulation is
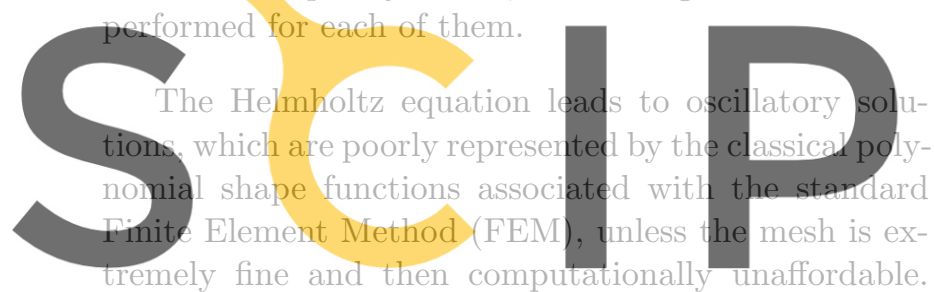

Moreover, for high wavenumber applications, the nu-

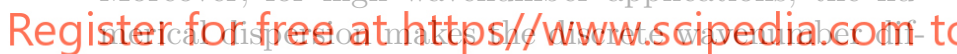

fer from that of the exact solution. This effect is also called pollution error, and separates the solution of the standard FEM from the best approximation [13] [14, Sect. 4.6] [15]. Alternative finite element formulations have been developed to overcome this shortcoming by including enriching functions into the approximation space. These enriching functions include a priori knowledge of the solution and provide better local approximation properties. This also improves the accuracy of the global approximation. In the case of the Helmholtz equation, it is advantageous to include sets of plane waves propagating in different directions [16-18], which form $c$-complete sets of functions for this equation [19]. Plane waves are free-space natural solutions if a uniform wavenumber is considered, and practically eliminate the pollution error [18]. In addition, this alleviates the constraint of having a minimum number of elements per wavelength (typically 10 or 12 as a rule of thumb), allowing the use of coarser meshes with several wavelengths per element, and providing a considerable reduction in the total number of unknowns (more than $90 \%$ in some cases [20]).
Enriched formulations have been recently incorporated to several approaches for the solving of the Helmholtz equation [21]. The Ultra-Weak Variational Formulation (UWVF) and the Discontinuous Enrichment Method (DEM) are examples of discontinuous enriched methods in which continuity between elements is weakly imposed. In the UWVF the Helmholtz equation is decomposed into coupled subproblems for each element $[22,23]$, while in the DEM continuity is weakly enforced by means of Lagrange multipliers [24,25]. Here, we follow the Partition of Unity Method (PUM) with plane waves, which is based on the partition of the unity theory $[16,26,17,18]$. It combines the good local approximation properties of the plane waves with the standard polynomial shape functions, that ensure continuity between elements.

The PUM has been extensively applied to diffraction and scattering problems [27-32, 20,33-35]. However, in this work we explore a novel application of the method by developing a numerical model to simulate the underwater noise propagation. The seawater absorption phenomenon is included through the imaginary part of the wavenumber. Our model considers non-

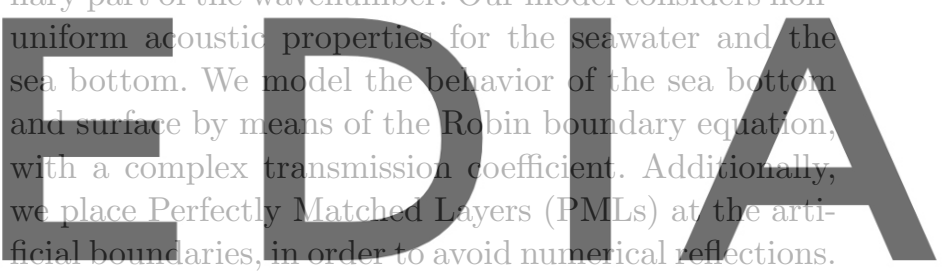

The introduction of plane waves in the basis of the

\section{downitodion thecversiops witkoute ther watermark}

the number of degrees of freedom of the problem, thanks to the oscillatory behavior of the functions. Nevertheless, this provokes an undesirable rise in the CPU time required for the integration step, if a standard GaussLegendre rule is selected, as in the first implementations of the method $[28,30,31,20,33]$. The reason is that a large number of integration points is required to capture the highly oscillatory integrands. Ortiz introduced a local coordinate rotation in order to develop a problemadapted semi-analytical integration rule $[29,32]$. Later, Strouboulis and Hidajat applied the Filon's rule to evaluate the integrals over rectangular elements [35]. In this work, we follow the semi-analytical approach developed by Bettes, Sugimoto et al., which is valid for first order triangular and quadrilateral elements $[36,37]$.

Several authors have reported ill-conditioning of the resulting matrices when sets of plane waves are considered as part of the approximation functions [16-18,27, $29,30,20,21]$. In order to reduce the condition number of the elemental matrices, we perform a low-rank approximation of the local basis functions. To this end, we first compute the volume integrals of the weak form 
over a standard domain patch. Then, we compute the singular values of the corresponding matrix, and truncate the smallest ones. This contribution improves the conditioning of the system matrix and reduces the number of degrees of freedom and, hence, the required computational resources.

The remainder of this paper is organized as follows. In Sect. 2 we introduce our physical model, setting up the problem and detailing how the physical phenomena are considered. In Sect. 3 we focus on the numerical formulation. Specifically, we detail the semi-analytical integration procedure used to compute the elemental contributions, and a technique providing a low-rank approximation based on the singular value decomposition (SVD). Several numerical example are presented in Sect. 4, to illustrate the applicability and accuracy of the proposed method. Finally, Sect. 5 includes some concluding remarks and developments to be addressed in the near future.

2 Modelization of the underwater noise propagation

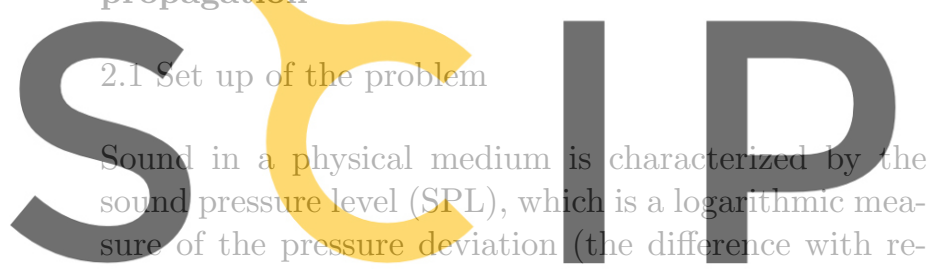

spect to the hydrostatic pressure in the case of underRegister for free, at https/ / Ww deviation is. going to

$\mathrm{SPL}=10 \log _{10}\left(\frac{p_{\mathrm{rms}}^{2}}{p_{0}^{2}}\right)=20 \log _{10}\left(\frac{p_{\mathrm{rms}}}{p_{0}}\right)$

where SPL is measured in decibels, relative to a reference pressure $p_{0}$ ( $1 \mu \mathrm{Pa}$ for underwater media, by convention), and $p_{\text {rms }}$ is the time-averaged root mean square pressure. For harmonic waves, $p_{\mathrm{rms}}=|p| / \sqrt{2}$, where $|p|$ is the modulus of the complex pressure $p$.

The speed of ocean waves and water masses are both small (around several meters per second) compared to the sound speed in water (approximately $1500 \mathrm{~m} / \mathrm{s}$ ). Hence, our model neglects water motion and assumes a completely still medium [38] and a still and plane sea surface, see Sect. 2.4. Accordingly, acoustics and fluid dynamics are uncoupled.

For the European offshore wind farms the characteristic water depth varies between $20 \mathrm{~m}$ and $200 \mathrm{~m}$, depending on the structural type of the turbine, while their characteristic length scale is a few kilometers [39]. Thus, the overall shape of the domain is nearly rectangular, with the horizontal dimension much larger than the vertical one. In our bi-dimensional model, the domain consists of an area enclosed by the sea surface (assumed planar), two artificial lateral boundaries, and the sea bottom, which at the working scale is usually smooth with slight slopes.

We assume stationary harmonic noise sources. In practice, input noise spectra are decomposed into a set of frequency bands, each of them characterized by a reference frequency (and a corresponding wavenumber). Then, the Helmholtz equation can be considered to compute a numerical solution for each of the single frequency bands, independently. Finally a sound pressure level spectra distribution is generated after collecting the results for each band.

\subsection{Problem statement}

The unknown complex pressure, $p$, takes values in $\Omega$ and is the solution of the boundary value problem

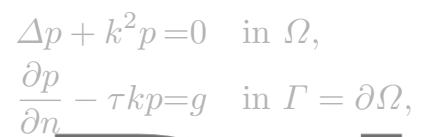

(2)

(3)
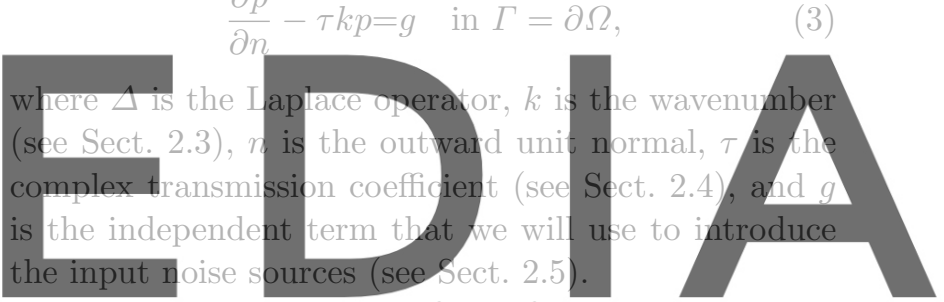

The corresponding weak form of the problem is stated as: find $p \in H^{1}(\Omega)$ such that

download the version without the watermark $\mathcal{B}(p, v)=\mathcal{L}(v) \quad \forall v \in H^{1}(\Omega)$,

where

$$
\begin{aligned}
\mathcal{B}(p, v) & =\int_{\Omega}\left(k^{2} p \bar{v}-\nabla p \cdot \nabla \bar{v}\right) \mathrm{d} \Omega+\int_{\Gamma} \tau k p \bar{v} \mathrm{~d} \Gamma \\
\mathcal{L}(v) & =-\int_{\Gamma} g \bar{v} \mathrm{~d} \Gamma,
\end{aligned}
$$

being $H^{1}(\Omega)$ the space of functions with square-integrable derivatives in $\Omega$, and · the conjugate of a complex variable.

\subsection{Seawater absorption and complex wavenumber}

The geometrical spreading, that is the attenuation of the intensity when the wave propagates away from the acoustic source, is implicitly accounted for in the elliptic nature of the Helmholtz equation. However, empirical evidence reveals that seawater is a damping medium. The additional attenuation associated with physicalchemical reactions is caused by two groups of phenomena that convert acoustic energy into heat: the shear 
and volume viscosities effects, and a series of ionic relaxation processes involving, mainly, magnesium sulfate $\mathrm{MgSO}_{4}$ and boric acid $\mathrm{B}(\mathrm{OH})_{3}$. The energy loss is modeled with the logarithmic absorption coefficient $\alpha$, which is estimated using the Ainslie and McColm formula [40].

This is accounted for in our model by considering a complex wavenumber $k=k_{1}+i k_{2}, i=\sqrt{-1}$. The imaginary part, $k_{2}$, introduces an omni-directional attenuation of the solution that is proportional to the absorption coefficient $\alpha$, namely

$k_{2}=\frac{\ln 10}{20 \cdot 1000} \alpha$,

where $k_{2}$ is measured in $\mathrm{rad} / \mathrm{s}$ and $\alpha$ in $\mathrm{dB} / \mathrm{km}$, see Appendix A for more details.

The real part of the wavenumber is standard: $k_{1}=$ $2 \pi f / c$, being $f$ the wave frequency and $c$ the sound speed. In the particular case of the seawater, $c$ depends on the temperature, the salinity and the hydrostatic pressure. These magnitudes are generally horizontally stratified, except in areas located near river mouths or suffering strong ice melting, and they are well described

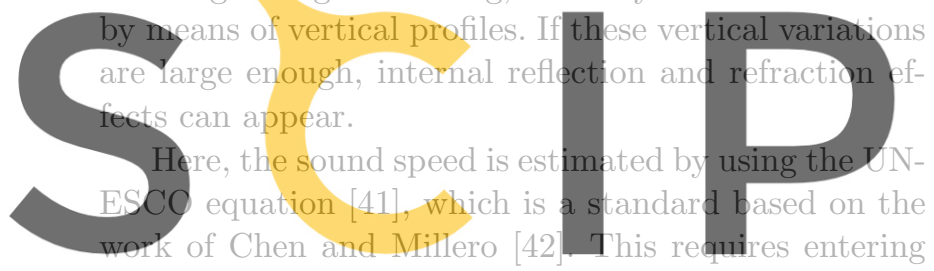

the salinity, the temperature and the hydrostatic pres-

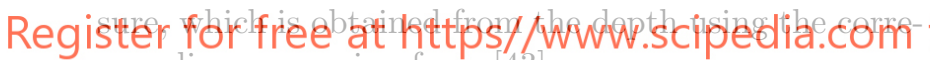
sponding expression from [43].

\subsection{Boundary conditions}

The boundary condition, Eq. (3), models the behavior of the solution at different parts of the boundary (sea surface, sea bottom, and lateral artificial boundaries). The dimensionless transmission coefficient $\tau$ is related to the rate of transfer of energy at the corresponding boundary [44], and it allows prescribing fully reflecting $(\tau=0)$, non-reflecting $(|\tau|=1)$ or partially reflecting $(0<|\tau|<1)$ boundary conditions [45].

Depending on the availability of empirical measurements, the value of the transmission coefficient can be obtained either from the transmission loss at the interface $\mathrm{TL}_{i}$, measured in $\mathrm{dB}$, or from the acoustic impedance of the boundary $Z_{b}=\rho_{b} c_{b}$, being $\rho_{b}$ the density of the boundary material, see Appendix B for more details. In the first case, we have

$\tau=i \frac{1-10^{-\mathrm{TL}_{i} / 20}}{1+10^{-\mathrm{TL}_{i} / 20}}$.
In the second case, we have

$\tau=i \frac{Z_{s w}}{Z_{b}}$,

where $Z_{s w} \approx 1.54 \times 10^{6} \mathrm{~kg} /\left(\mathrm{m}^{2} \mathrm{~s}\right)$ is the acoustic impedance of the seawater.

Note that we can reproduce full reflection (Neumann boundary conditions) by setting $\tau=0\left(\mathrm{TL}_{i}=0\right.$ or $Z_{b}>>Z_{s w}$ ), and null reflection (0-order absorbing boundary conditions) by setting $\tau=i\left(\mathrm{TL}_{i} \rightarrow \infty\right.$ or $\left.Z_{s w}=Z_{b}\right)$.

The sea surface behaves as a reflector when it is smooth, due to the large acoustic impedance contrast, and as a scatterer when it is rough. The sea surface is treated as plane and fully reflective $(\tau=0)$, being a conservative assumption with respect to all possible sea states.

On the contrary, the impedance mismatch between the seawater and sea bottom materials is less severe and, hence, the sea bottom is considered as a partially reflecting and scattering boundary. Its acoustic properties depend, for instance, on the multi-layered composition of the materials, the frequency and the angle

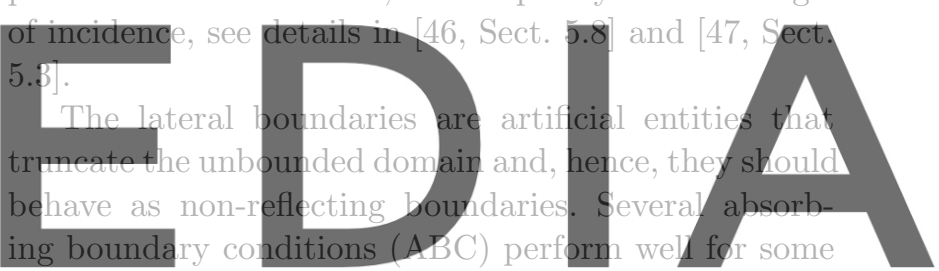

propagation directions, but introduce spurious reflections for others. Higher order ABCs are more accudownload the version without the watermark of storage and calculation time [48]. Here, Perfectly Matched Layers (PNiLs) are adopted in order to achieve full absorbing conditions and avoid spurious reflections [49]. PMLs are analytic continuations of the Helmholtz equation into complex spatial coordinates, see [50]. The PML technique assumes homogeneous medium properties along the normal to the boundary. In order to simplify the formulation, the lateral boundaries are taken parallel to the axes.

\subsection{Noise generation}

Note that the size of the sound sources (wave energy generators, wind turbines, or other elements) is small compared to the size of the domain. Moreover, the region of interest is usually placed at distances equal to several times their characteristic size. Hence, we assume that noise sources are punctual and located on the surface.

The input noise is introduced via the analytical solution of the pressure field generated by a point source 
with the hypothesis of a uniform wavenumber $k_{0}$. This pressure field is introduced into the Robin boundary equation (3) and, then, the resulting independent term $g$ is integrated along the boundary edges of the adjacent elements. Specifically, the pressure field $p_{a}$ produced by a point source in a homogeneous $2 \mathrm{D}$ medium can be approximated by (see [51, Chapt. 5])

$p_{a}(\boldsymbol{x})=p_{0}\left(\frac{r\left(\boldsymbol{x}_{\mathbf{0}}\right)}{r(\boldsymbol{x})}\right)^{1 / 2} \exp \left(i k_{0}\left(r(\boldsymbol{x})-r\left(\boldsymbol{x}_{\mathbf{0}}\right)\right)\right)$,

where $\boldsymbol{x}$ is the spatial coordinates vector, $p_{0}$ is the pressure produced by the source at a reference point $\boldsymbol{x}_{\mathbf{0}}$, and $r(\cdot)$ is the distance to the point source $\boldsymbol{x}_{s}$. Hence, according to Eq. (3),

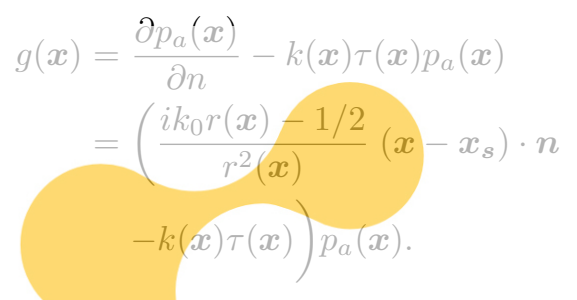

Note that the source point must be separated from the surface since there is a singularity point at $r\left(x_{s}\right)=0$.

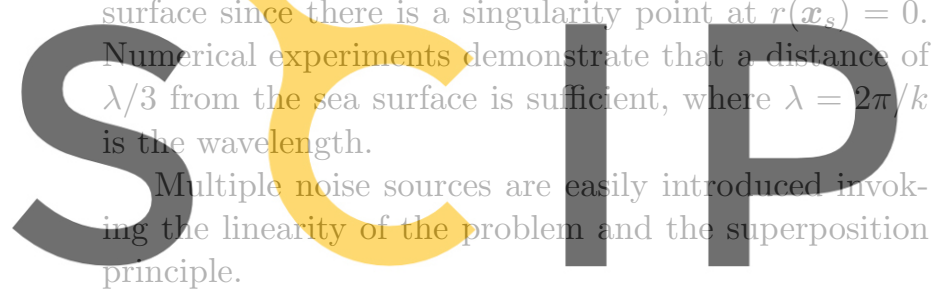

Register for free at https//www.scipedia.com 3 Numerical model

\subsection{Partition of Unity Method}

The boundary value problem defined by Eq. (4) is solved using the Partition of Unity Method (PUM). Domain $\Omega$ is discretized using a quadrilateral mesh with characteristic size $h$, see Sect. 3.2, and a total number of nodes $n_{\text {nod }} . N_{m}$, for $m=1, \ldots, n_{\text {nod }}$, denotes the classical piecewise bilinear shape function associated to the $m$-th node of the mesh. The support of $N_{m}, \nu_{m}$, is a patch composed by the elements sharing node $m$.

The partition of unity space $\mathcal{W}_{h, q}$ reads

$\mathcal{W}_{h, q}=\left\{v \mid v=\sum_{m=1}^{n_{\text {nod }}} N_{m}\left(\sum_{r=1}^{q} a_{m, r} W_{m, r}\right)\right\}$,

where $q$ is the number of plane waves (enrichment functions) pasted at the $m$-th node (patch), $a_{m, r}$ is the complex coefficient (amplitude) of the $r$-th plane wave associated to the $m$-th node, namely

$W_{m, r}(\boldsymbol{x})=\exp \left(i k_{m} \boldsymbol{e}_{\boldsymbol{r}} \cdot\left(\boldsymbol{x}-\boldsymbol{x}_{\boldsymbol{m}}\right)\right)$,
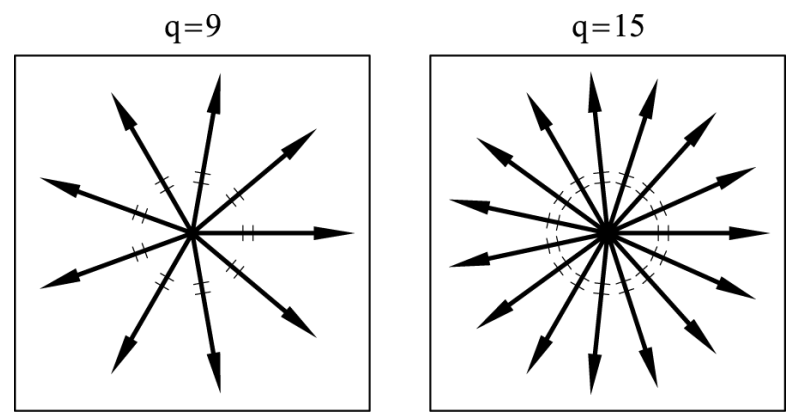

Fig. 1: Examples showing possible directions of the 2D plane waves enriching functions employed at each node

being $k_{m}$ the wavenumber at the $m$-th node, $\boldsymbol{e}_{\boldsymbol{r}}=$ $\left(\cos \left(\theta_{r}\right), \sin \left(\theta_{r}\right)\right)$ the $r$-th propagation direction, $\theta_{r}=$ $(2 \pi r) / q$ the counterclockwise angle with respect to the $x$ axis, and $x_{m}$ the coordinates of the $m$-th node. Figure 1 depicts two examples of sets with equidistributed directions for the plane waves.

\section{Remark 1 Functions $N_{m}$, for $m=1, \ldots, n_{\text {nod }}$, are a partition of the unity}

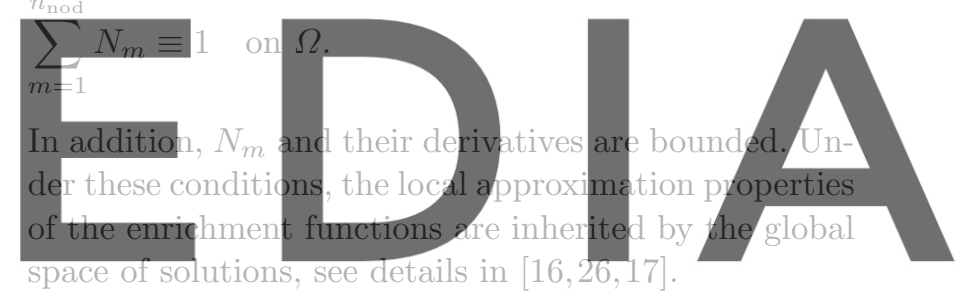

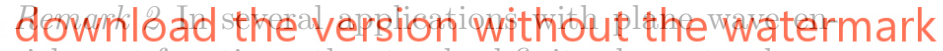
richment functions, the standard finite element polynomial approximation space is kept in the definition of the solution space $[52,53]$. That is, Eq. (12) is replaced by

$$
\begin{aligned}
\mathcal{W}_{h, q}=\{v \mid v= & \sum_{m=1}^{n_{\text {nod }}} b_{m} N_{m} \\
& \left.+\sum_{m=1}^{n_{\text {nod }}} N_{m}\left(\sum_{r=1}^{q} a_{m, r} W_{m, r}\right)\right\} .
\end{aligned}
$$

Here, we adopt the approach presented in [17,27-30, $32,33,35]$ that only considers the part of the approximation space including the planar waves (we assume $b_{m}=0$ in Eq. (14)). This is supported by two reasons. First, the enhanced rate of convergence is given by the contribution of the plane wave basis, rather than the polynomials [32]. Second, we deal with pressure fields that a priori have null average.

Remark 3 Although it is possible to use high-order finite element shape functions, we consider bilinear polynomials, following the rationale of Remark 2. Moreover, 
this also simplifies the semi-analytical integration rules to compute the elemental contributions.

Remark 4 The definition of the enrichment functions (13) is local. Thus, it is possible to paste different sets of plane waves at each node patch. We take advantage of this property to incorporate non-uniform distributions for the sound speed (wavenumber). The wavenumber evaluated at the $m$-th node is assigned to the plane waves pasted at the $m$-th patch. Note that the $k_{m}$ value applies to the whole patch, thus, it is assumed that the spatial gradient of the sound speed is smooth.

The PUM solution of the weak problem, Eq. (4), is defined as follows. Find $p_{h, q} \in \mathcal{W}_{h, q}$ such that
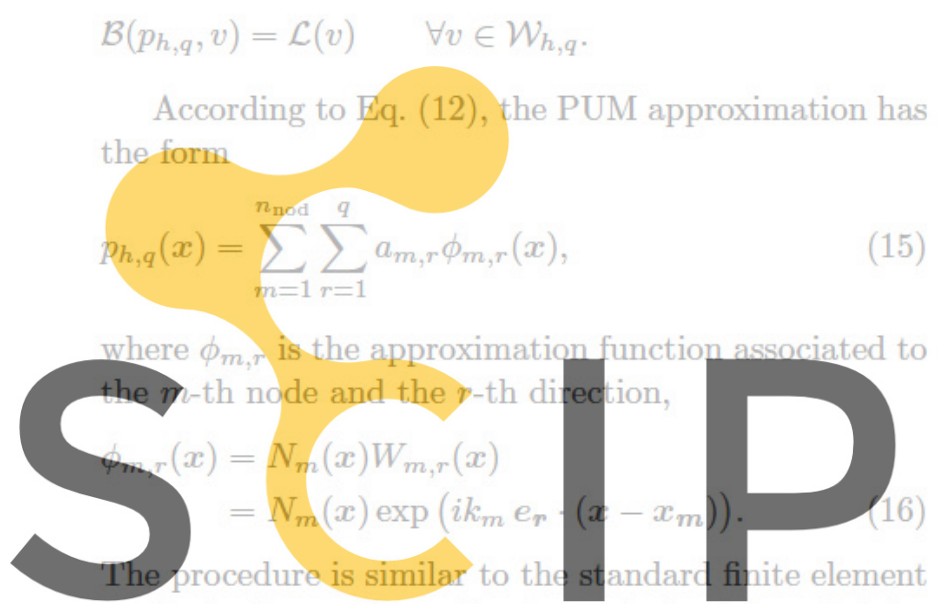
method scheme. Here, the terms to be integrated include harmonic functions and, therefore, the correspondRegister for free at https/dwwW. Scipedia. com to

ing specific semi-analytical integration rules (see Sect. 3.3), and assembled in the global matrix and right-hand side vector. Then, the algebraic complex linear system is solved numerically. Finally, a specific post-processing has to be efficiently applied on the resulting solution vector to recover the pressure field, Eq. (15).

\subsection{Domain discretization}

The computational domain is the sea area enclosed by 3 straight segments (the planar sea surface and 2 lateral artificial boundaries defined by the PML layers) and a smooth sea bottom curve. Therefore, the domain is quasi-rectangular and can be easily discretized using quadrilateral elements. First, a preliminary structured grid of rectangles is generated. Second, the lower rows of elements are adapted to fit the bottom. Thus, the resulting meshes are composed of 3 types of elements (see Fig. 2): rectangular geometrically identical non-PML elements (white elements), quadrilateral non-PML elements (gray elements), and rectangular PML elements

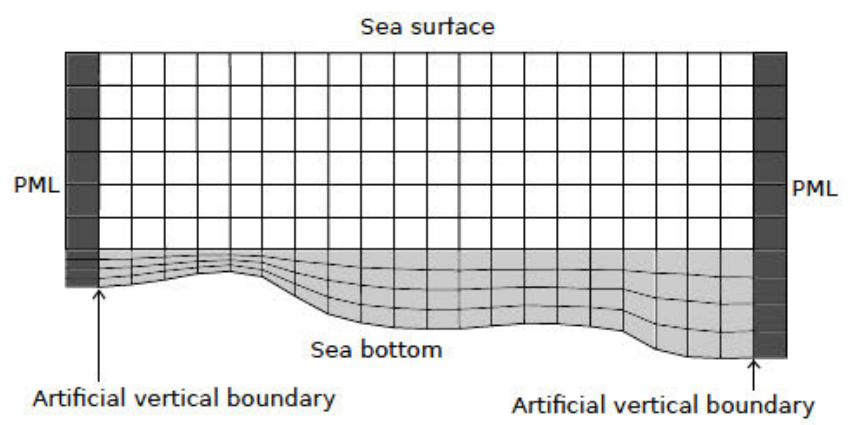

Fig. 2: Example of a two-dimensional mesh of quadrilaterals, with PMLs at the artificial vertical boundaries

(dark gray elements). We will take advantage of theses structured meshes during the computation of the integrals involved in the element contributions of the weak form (4), see Sect. 3.3.

Two factors determine the target element size $h$ : the number of waves per element and the characteristic bathymetric length, that is, the length associated to the roughness of the sea bottom definition. For lowfrequency simulations (below approximately $100 \mathrm{~Hz}$ ).

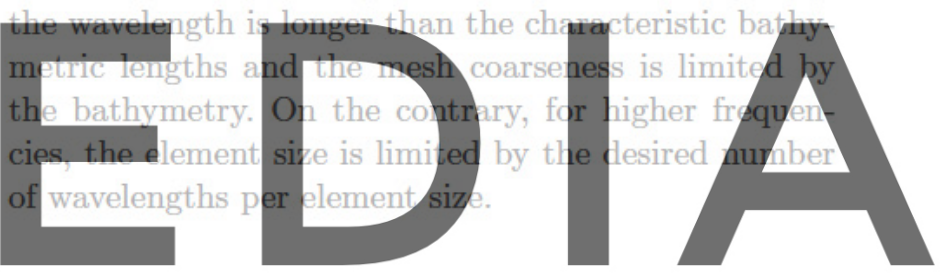

3.3 Numerical integration

download the version without the watermark The approximation functions proposed by the PUM enriched with plane waves are adapted to the nature of the solution. Thus, the enriched approximation space allows a drastic reduction of the pollution error, see [13] and [14, Sect. 4.8] for a detailed analysis. The number of degrees of freedom is also reduced because the same accuracy is reached with coarser meshes. However, due to the highly oscillatory behavior of the resulting integrands, a high number of integration points (up to $120 \times 120$ points $[28,33])$ is needed to compute the elemental contributions, if a standard Gauss-Legendre rule is applied. Therefore, the integration cost becomes prohibitive if high frequencies or large domains are considered unless a more efficient quadrature, particularly devised for this case, is used.

In our model, three kinds of highly oscillatory integrals are evaluated in order to compute the element contributions. We classify them into two groups. In the first group, we have the integrals involved in the computation of the form $\mathcal{B}(p, v)$, see Eq. (5). These two integrals are finite Fourier transform type, with a smooth non-oscillatory part and a highly oscillatory complex 
exponential part. In the second group, we have the integral involved in the computation of the linear operator $\mathcal{L}(v)$, see Eq. (6), where the integrand is the product of two highly oscillatory terms.

In the first group of integrals, we have to evaluate the volume integral matrix (the first term in Eq. (5)). The contribution of the $e$-th element is

$$
\begin{aligned}
K_{m r, n s}^{(e)}= & \int_{\Omega^{(e)}}\left[k^{2} \phi_{n, s} \bar{v}_{m, r}-\nabla \phi_{n, s} \cdot \nabla \bar{v}_{m, r}\right] \mathrm{d} \Omega \\
= & \exp \left[i\left(\bar{k}_{m} \boldsymbol{e}_{\boldsymbol{r}} \cdot \boldsymbol{x}_{\boldsymbol{m}}-k_{n} \boldsymbol{e}_{\boldsymbol{s}} \cdot \boldsymbol{x}_{\boldsymbol{n}}\right)\right] \\
& \int_{\Omega^{(e)}} F_{m r, n s} \psi_{m r, n s} \mathrm{~d} \Omega
\end{aligned}
$$

where $\phi_{n}^{s}$, and $v_{m}^{r}$ are defined according to Eq. (16), $m, n=1, \ldots, n_{\text {nod }}$, and $r, s=1, \ldots, q$,
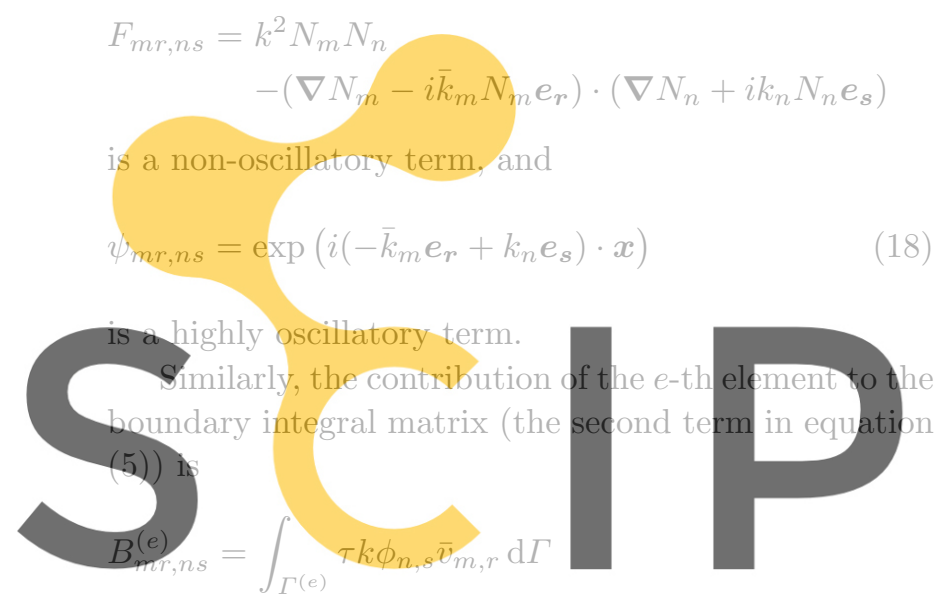

$=\exp \left[i\left(\bar{k}_{m} e_{r} \cdot x_{m}-k_{n} e_{s} \cdot x_{n}\right)\right.$ $\int_{\Omega^{(e)}} F_{m r, n s} \psi_{m r, n s} \mathrm{~d} \Gamma$,

where

$F_{m n, r s}=\tau k N_{m} N_{n}$

is the non-oscillatory term and $\psi_{m r, n s}$ is given by Eq. (18).

To compute the integrals in Equations (17) and (19) we have to evaluate expressions of the type

$I=\int_{\boldsymbol{X}} F_{m r, n s}(\boldsymbol{x}) \psi_{m r, n s}(\boldsymbol{x}) \mathrm{d} \boldsymbol{X}$

where $\boldsymbol{X}$ is a $1 \mathrm{D}$ or a $2 \mathrm{D}$ domain. To this end, we use the semi-analytical quadratures developed by Bettess et al. $[36,37]$. In these rules, the non-oscillatory term of the integrands is approximated by a set of $n_{\mathrm{lp}}$ Lagrangian polynomials,

$F_{m r, n s}(\boldsymbol{x}) \approx \sum_{p=1}^{n_{\mathrm{lp}}} F_{m r, n s}\left(\boldsymbol{x}_{p}\right) L_{p}(\boldsymbol{x})$, where $\boldsymbol{x}_{p}, L_{p}(\boldsymbol{x})$ are the $p$-th interpolation point and its associated Lagrange polynomial, respectively. Therefore, we have

$$
\begin{aligned}
I & =\sum_{p=1}^{n_{\mathrm{lp}}} F_{m r, n s}\left(\boldsymbol{x}_{p}\right) \int_{\boldsymbol{X}} L_{p}(\boldsymbol{x}) \psi_{m r, n s}(\boldsymbol{x}) \mathrm{d} \boldsymbol{X} \\
& =\sum_{p=1}^{n_{\mathrm{lp}}} F_{m r, n s}\left(\boldsymbol{x}_{p}\right) w_{m r, n s ; p}
\end{aligned}
$$

where

$w_{m r, n s ; p}=\int_{\boldsymbol{X}} L_{p}(\boldsymbol{x}) \psi_{m r, n s}(\boldsymbol{x}) \mathrm{d} \boldsymbol{X}$.

are the integration weights. The key point in the rules proposed by Bettess et al. [36,37] is to perform an analytical integration for these integration weights.

Hence, the quality of the integrals (17) and (19) is determined by the quality of the approximation (20). Note that the non-oscillatory functions are evaluated at the interpolating points, whose spacing is determined by the behavior of this functions rather than by the highly oscillatory full integrand, resulting in a moderate amount of points and function evaluations, com-

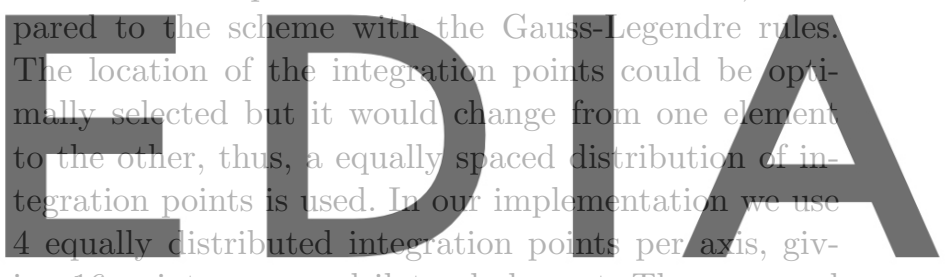

ing 16 points per quadrilateral element. The proposed

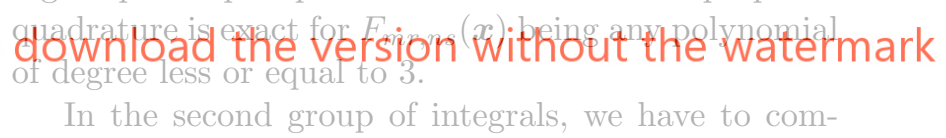
pute the element contribution of the independent term $g$ of the Robin equation (noise generation) along the boundary edges surrounding the noise sources, see Eq. (11),

$$
\begin{aligned}
f_{m r}^{(e)}= & \int_{\Gamma^{e}} g \bar{v}_{m, r} \mathrm{~d} \Gamma \\
= & \exp \left(i \bar{k}_{m} \boldsymbol{e}_{\boldsymbol{r}} \cdot \boldsymbol{x}_{\boldsymbol{m}}\right) \\
& \int_{\Gamma^{e}} \underbrace{g(\boldsymbol{x}) N_{m}(\boldsymbol{x})}_{\text {highly oscillatory }} \underbrace{\exp \left(-i \bar{k}_{m} \boldsymbol{e}_{\boldsymbol{r}} \cdot \boldsymbol{x}\right)}_{\text {highly oscillatory }} \mathrm{d} \Gamma^{(e)} .
\end{aligned}
$$

Since function $g$ is highly oscillatory the semi-analytical methods previously proposed are less competitive (the computational cost involved in the evaluation of a large number of integration weights (21) is high). Consequently, we use a standard high order Gauss-Legendre rule to compute Eq. (22) using 10 integration points per wavelength.

Remark 5 The integration step in the PUM has a larger impact in the CPU time, with respect to the standard 
FEM. The computational cost of the integration of the volume integrals $K_{m r, n s}^{(e)}$ can be reduced if the geometrical properties of the domain discretization are considered. Since each layer of the upper region of the mesh is composed of elements that are geometrically identical, and if the physical parameters of the elements are the same (sound speed and absorption coefficient), the elemental matrices can be reused for several elements, reducing drastically the $\mathrm{CPU}$ time involved in the simulation.

3.4 Low-rank approximation of the local enrichment basis

It is well known that the element matrices, Eqs. (17) and (19) and, therefore, the corresponding global matrix obtained by the PUM method are ill-conditioned, when the solution space is enriched by a set of plane waves $[27,29,30]$. To mitigate this drawback, we devise a low-rank approximation of the local functional discretisation space discarding the redundant elements of the basis and keeping the relevant terms, associated with the highest singular values provided by the Sin-

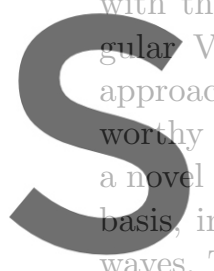
Talue Decomposition h, already mentioned to be explored, in [20], low-rank approximation in the context of the $\mathrm{P}$ The approximation is obta
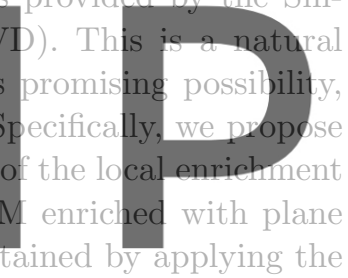

SVD to identify and truncate the smallest singular val-

Register for firee iatchttepsh/uwyw scilpedia.carn. to

This introduces a linear transformation of the local solution and local test spaces, leading to a new number $\tilde{q}$ of approximation functions per node, and reduces the size of the system of equations. As a consequence, a large saving in the computational cost (both in terms of CPU time and memory footprint) is produced.

The low-rank approximation is computed with the following procedure. First, a standard patch (set of elements sharing a node) is defined using the physical properties of a rectangular element of the upper rows of the discretization (white elements in Fig. 2), and a uniform sound speed is set as an average of the vertical sound speed profile. Then, the approximation functions associated with the patch (to the inner node of the patch) are integrated according to (17). The SVD is then applied to the resulting $q \times q$ matrix:

$\mathbf{K}^{\text {patch }}=\mathbf{U} \boldsymbol{\Delta} \mathbf{V}^{*}$,

where the $q \times q$ diagonal matrix $\Delta$ contains non-negative real numbers, the singular values of $\mathbf{K}^{\text {patch }}, \mathbf{U}, \mathbf{V}$ are $q \times q$ unitary matrices, and ${ }^{*}$ denotes conjugate transpose.
Remark 6 If seawater absorption is not considered, the wavenumber $k$ is real. Thus, matrix $\mathbf{K}^{\text {patch }}$ is hermitian, see Eq. (5). In this case $\mathbf{U}=\mathbf{V}$. However, for the general case, the wavenumber $\mathrm{k}$ is complex, and $\mathbf{K}^{\text {patch }}$ is no longer hermitian.

Let $\delta_{\max }=\max \left\{\delta_{i}\right\}$ for $i=1, \ldots, q$ be the $\max -$ imum singular value. We select those singular values that verify

$\frac{\delta_{i}}{\delta_{\max }} \geq \varepsilon \approx \frac{1}{\kappa\left(\mathbf{K}^{\text {patch }}\right)}$,

being $\varepsilon$ a given tolerance and $\kappa(\cdot)$ the condition number. We define the square reduced diagonal matrix $\tilde{\boldsymbol{\Delta}}_{\tilde{q} \times \tilde{q}}$, including the largest $\tilde{q}$ singular values of $\boldsymbol{\Delta}$, and the rectangular reduced matrices $\tilde{\mathbf{U}}_{(q \times \tilde{q})}$ and $\tilde{\mathbf{V}}_{(q \times \tilde{q})}$, composed by the corresponding $\tilde{q}$ columns of the matrices $\mathbf{U}$ and $\mathbf{V}$, respectively. Therefore, matrix $\mathbf{K}^{\text {patch }}$ is approximated by
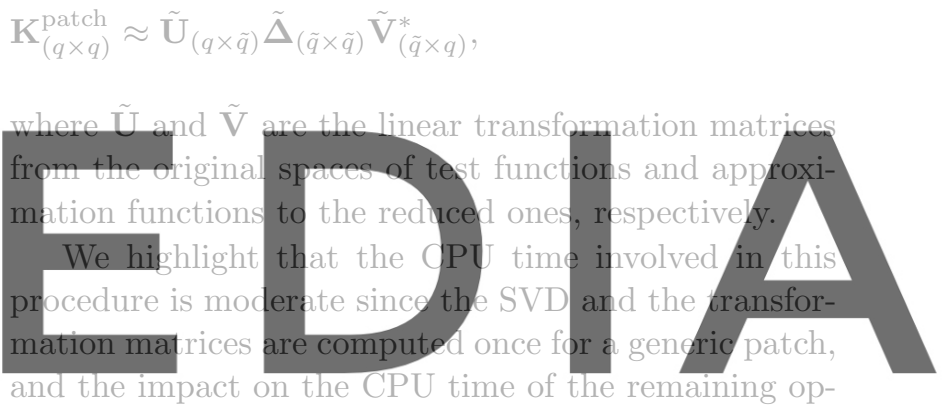
erations is negligible.

\section{downhloaf the version without thenwatermark}

trices and independent term vectors are integrated for the original approximation and test functions in order to take profit of the semi-analytical rules presented in Sect. 3.3. After that, the following transformations are applied over the nodal blocks $\mathbf{K}_{(m, n)}^{(e)}$ and $\boldsymbol{f}_{(m)}^{(e)}$, before the assembly:

$$
\begin{aligned}
\tilde{\mathbf{K}}_{(m, n)}^{(e)} & =\tilde{\mathbf{U}}^{*} \mathbf{K}_{(m, n)}^{(e)} \tilde{\mathbf{V}}, \\
\tilde{\boldsymbol{f}}_{m}^{(e)} & =\tilde{\mathbf{U}}^{*} \boldsymbol{f}_{m}^{(e)},
\end{aligned}
$$

for $m, n=1, \ldots, 4$, and $e=1, \ldots, n_{\text {elem }}$, being $n_{\text {elem }}$ the number of elements.

Once the reduced system is solved, the transformation of the space of solutions is reverted in order to apply the original post-processing routines,

$\boldsymbol{a}_{(m)}=\tilde{\mathbf{V}} \tilde{\boldsymbol{a}}_{(m)}$,

where $\boldsymbol{a}_{(m)}$ and $\tilde{\boldsymbol{a}}_{(m)}$ are the vectors containing the complex coefficients associated to the m-th node in the original ( $q$ components) and reduced ( $\tilde{q}$ components $)$ local approximation spaces, respectively. 


\section{Numerical examples}

In this section we present four examples that illustrate the capabilities and the behavior of the proposed method. First, we test our model by computing the free propagation (without the interaction of obstacles or boundaries) of the sound generated by a point source in a lossy medium. The second example analyzes the effect of having different sea bottom transmission coefficients on the computed pressure field. In the third example we compute the pressure field when the sound speed profile is non-uniform, and two noise sources are considered. Finally, we perform a sensitive analysis of the low-rank approximation of the local basis on the accuracy and the required computational resources.

In all the examples, the sea surface is assumed horizontal and fully reflective $(\tau=0)$. The noise sources generate a harmonic sound with an amplitude of 10 $\mathrm{Pa}(\mathrm{SPL}=137 \mathrm{~dB}$, see Eq. (1)) at a distance of 1 m. The attenuation parameter for the PMLs is equal to zero at the non-PML regions and grows $10 \mathrm{rad} / \mathrm{s}$ per wavelength at the PMLs. The local spaces of solutions contain an initial number of $q=200$ plane waves
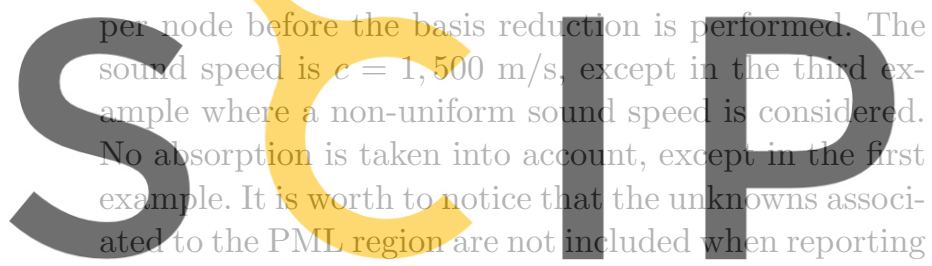

the total number of unknowns of each simulation. The

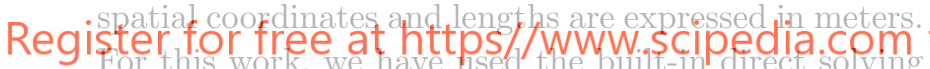
algorithm mldivide, included in MATLAB R2013b.

\subsection{Free propagation through a lossy medium}

The objective of this example is to illustrate the accuracy of the proposed model when the seawater absorption is taken into account. We consider the free propagation field generated by a single point source through a half-plane. The domain is truncated creating a rectangular geometry $\Omega=[-500,+500] \times[-100,0]$, and discretized using a rectangular mesh with 10 wavelengths per element, resulting in an element size of $(\Delta x, \Delta y)=(29.4,25)$, and giving 26,775 DOFs $(\tilde{q}=$ 153). The source is located at the center of the surface, $\boldsymbol{x}=(0,0)$, and generates a noise with a frequency of $f$ $=500 \mathrm{~Hz}$. A layer of PML elements is added around the lateral artificial boundaries and below the sea bottom boundary, see Fig. 3.

Two cases are analyzed. First, we consider the case with null seawater absorption $(\alpha=0 \mathrm{~dB} / \mathrm{km})$. Second, to highlight the effects of the attenuation, we set $\alpha=$

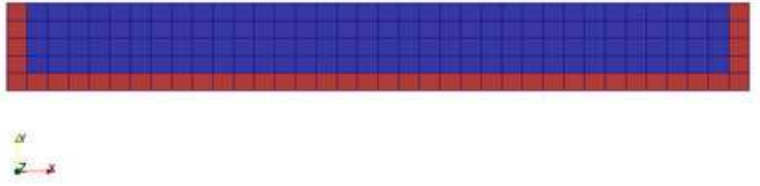

Fig. 3: 2D mesh used in Example 1, including non-PML elements (in blue) and PML elements (in red)

$50 \mathrm{~dB} / \mathrm{km}$, that in fact corresponds to the absorption produced at a higher frequency value of $100 \mathrm{kHz}$ (see Fig. 13 in Appendix A).

Figures $4 \mathrm{a}$ and $5 \mathrm{a}$ show the real part of the pressure field and the sound pressure level, when null absorption is considered. Note that the decay in the sound pressure level caused by geometrical spreading is clearly recognizable in Figure 5a. Figures 4b and 5b depict the same fields when the seawater absorption is considered. As expected, the solution includes an additional attenuation with respect to the solution without absorption. The additional attenuation associated with the seawater absorption is illustrated in Figures 4c and 5c plot-

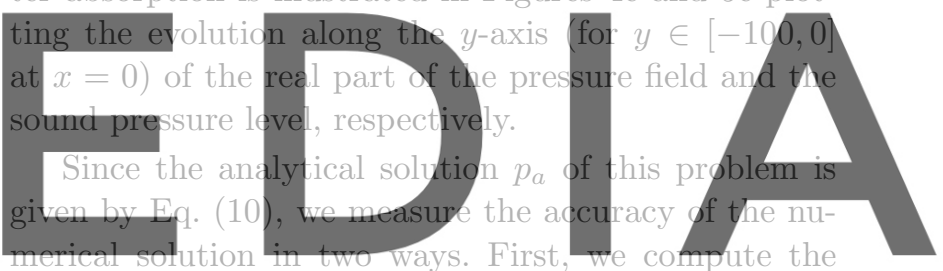

point-wise relative error as the discrepancy with the

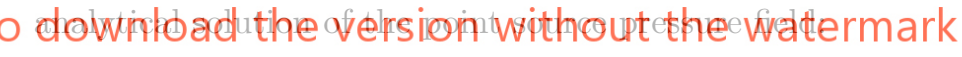

$\varepsilon_{\mathrm{pw}}(\boldsymbol{x})=\frac{\left|p_{h, q}(x)-p_{a}(x)\right|}{\left|p_{a}(\boldsymbol{x})\right|}$

The maximum value of this relative error remains below 25\%. Fig. 6 shows this value in both cases. From this figure we realize that the highest values of the point-wise relative error are located in two regions. On the one hand, they are located near the sea surface and far of the input noise sources. This may be caused by the method used to introduce the input noise, since we integrate the independent term of the Robin equation along the boundary edges of few elements around the noise source, see Sect. 2.5. On the other hand, large errors are also located along the edges of the quadrilateral elements.

Second, we also measure the accuracy of the PUM solution by computing the relative error in $L^{2}$ norm as

$\varepsilon_{L^{2}}=\frac{\left\|p_{h, q}(\boldsymbol{x})-p_{a}(\boldsymbol{x})\right\|_{L^{2}}}{\left\|p_{a}(\boldsymbol{x})\right\|_{L^{2}}}$ 

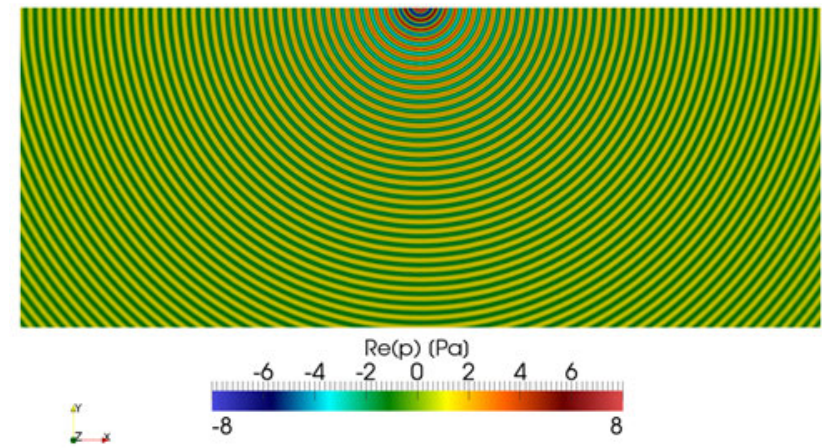

(a)
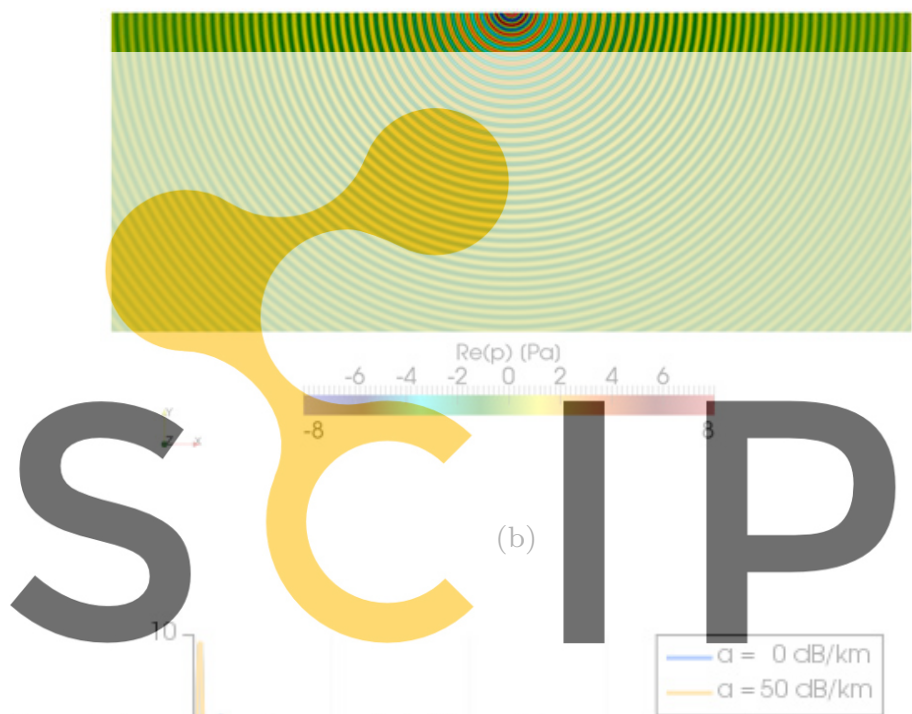

Registes for free at https//www.scipedia.com to

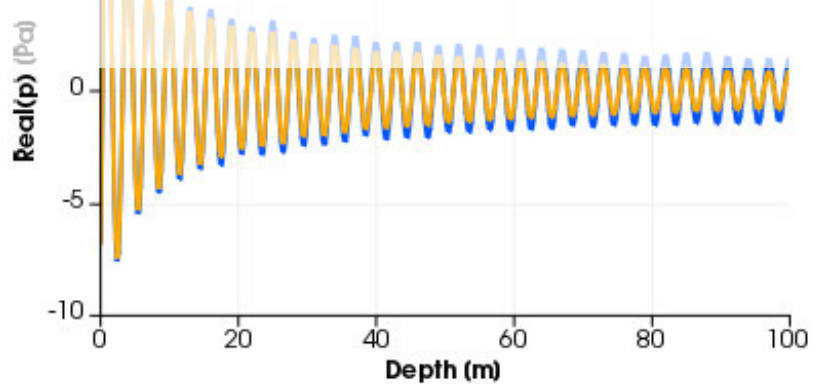

(c)

Fig. 4: Detail of the real part of the pressure at the central region of the domain $(x \in[-125,+125])$ in Example 1: (a) case without absorption; and (b) case with absorption. (c) Real part of the pressure field along the $\mathrm{y}$ axis for both absorption values

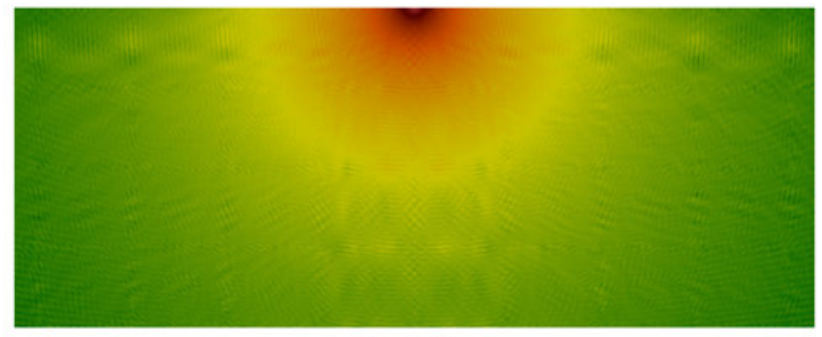

SPL (dB)

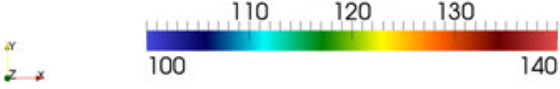

(a)
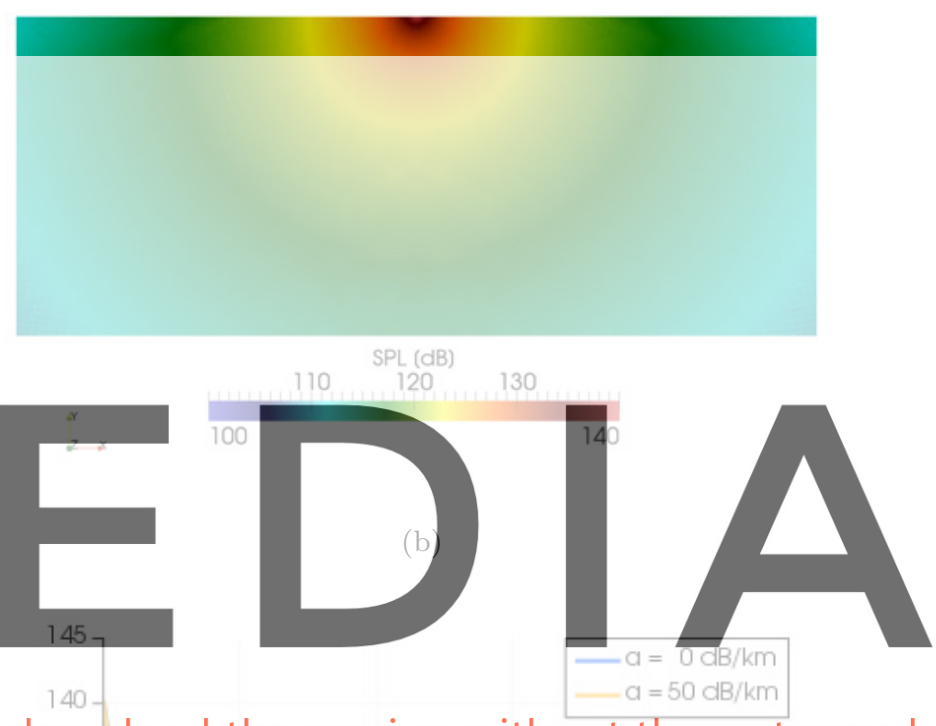

$140-1-a=50 \mathrm{~dB} / \mathrm{km}$

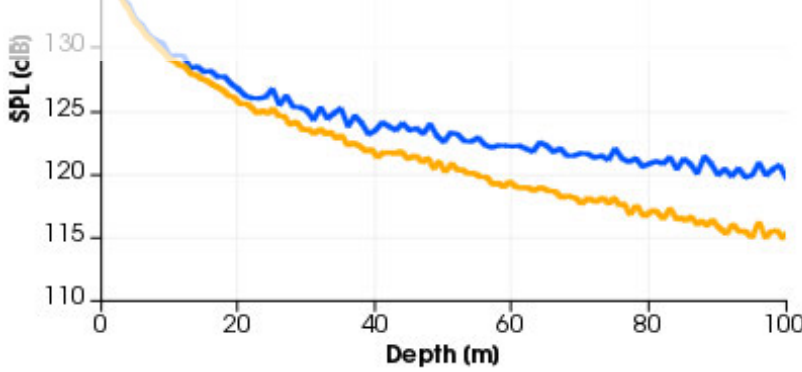

(c)

Fig. 5: Detail of the sound pressure level $(\mathrm{dB})$ at the central region of the domain $(x \in[-125,+125])$ in Example 1: (a) case without absorption; and (b) case with absorption. (c) SPL distribution along the y axis for both absorption values 


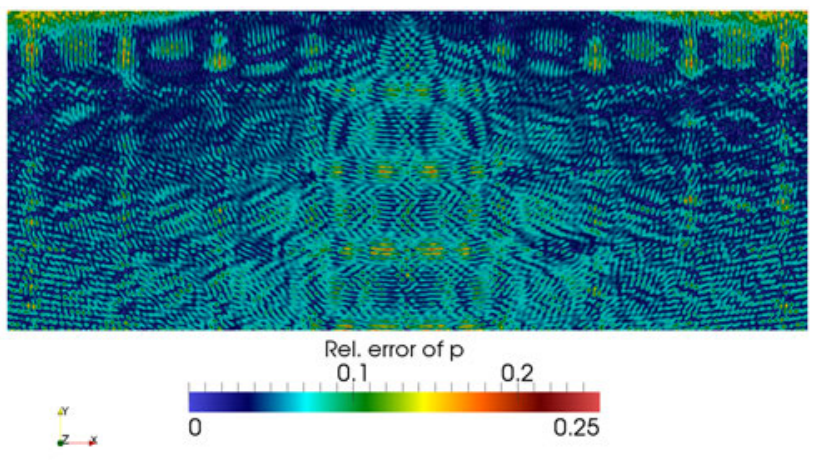

(a)
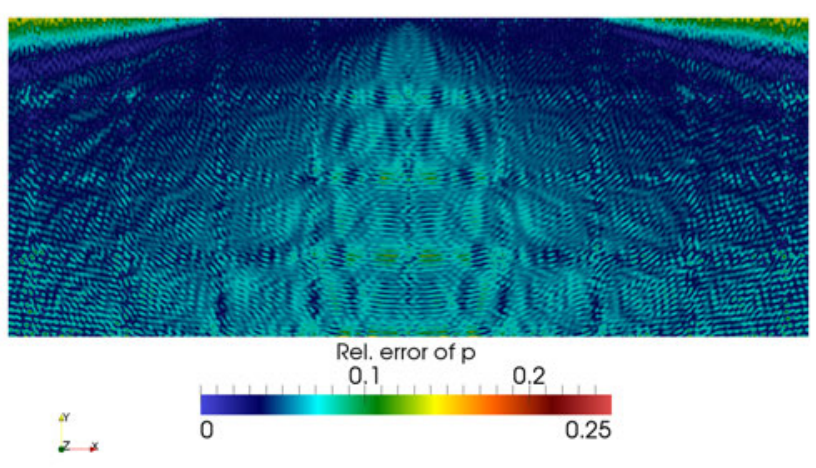

(b)

Fig. 6: Detail of the point-wise relative error in the pressure at the central region of the domain $(x \in$ $[-125,+125])$ in Example 1: (a) case without absorption; and (b) case with absorption

where $p_{h, q}(\boldsymbol{x})$ is the numerical solution, and

$\|\cdot\|_{L^{2}}=\sqrt{\int_{\Omega}|\cdot|^{2} \mathrm{~d} \Omega}$,

being $|\cdot|$ the complex modulus.

We obtain $\varepsilon_{L^{2}}=0.1120$ and $\varepsilon_{L^{2}}=0.0541$, for the lossless and lossy cases, respectively. In order to assess the relevance of these error values and if they are acceptable for engineering applications, it is important relating them to the standard relative errors of a quantity in environmental underwater acoustics such as the SPL. To this end, let $E_{\mathrm{SPL}}$ be the absolute error in the SPL generated by an absolute error, $E_{p}$, in the pressure field. Thus, from Eq. (1) and according to the Taylor expansion, we have

$$
\begin{aligned}
\mathrm{SPL}+E_{\mathrm{SPL}} & =20 \log _{10}\left(\frac{p+E_{p}}{p_{0}}\right) \\
& =20 \log _{10}\left(\frac{p}{p_{0}}\right)+\frac{1}{\ln (10)} \frac{E_{p}}{p}+\mathcal{O}\left(E_{p}^{2}\right) .
\end{aligned}
$$
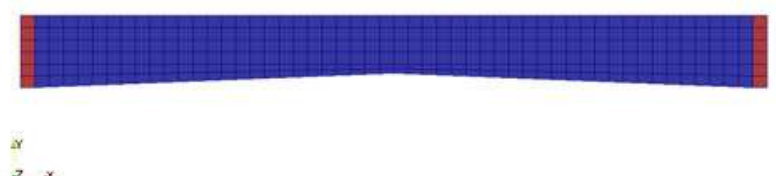

Fig. 7: 2D mesh used in Example 2, including non-PML elements (in blue) and PML elements (in red)

Thus, the same expression for the relative errors reads $\varepsilon_{\mathrm{SPL}} \mathrm{SPL}_{\mathrm{ref}} \approx \frac{1}{\ln (10)} \varepsilon_{p}$,

where $\varepsilon_{\mathrm{SPL}}=E_{\mathrm{SPL}} / \mathrm{SPL}_{\text {ref }}$ being $\mathrm{SPL}_{\text {ref }}$ a reference $\mathrm{SPL}$ value, and $\varepsilon_{p}=E_{p} / p$. The SPL in standard audiograms of marine mammals and other fish species ranges from $30 \mathrm{~dB}$ to $150 \mathrm{~dB}$ [3], and is usually given with two significant digits i.e. $\varepsilon_{\mathrm{SPL}} \leq \frac{1}{2} 10^{-2}$. Therefore, the worst case scenario corresponds to $\mathrm{SPL}_{\mathrm{ref}}=30 \mathrm{~dB}$ leading to

$\varepsilon_{p}=\ln (10) \mathrm{SPL}_{\mathrm{ref}} \varepsilon_{\mathrm{SPL}}=0.34$.

Therefore, the obtained values for point-wise relative error (24), and for the global relative error (25) remain inside typical engineering acceptable limits.

\subsection{Sea bottom with different transmission coefficients}

This example includes a bottom composed of two different sloped segments. The left-hand side (LHS) segment has a slope of +0.04 and a transmission coefficient $\tau=0.9 i$ ('soft' material, low impedance mismatch), while the right-hand side (RHS) segment has a slope of -0.04 and a transmission coefficient of $\tau=0.1 i$ ('hard' material, high impedance mismatch). The domain depth varies between $100 \mathrm{~m}$ at both lateral ends $(x=-500 \mathrm{~m}$ and $x=500 \mathrm{~m})$, and $80 \mathrm{~m}$ at the center of the domain, under the noise source. We consider the propagation of the sound generated by a single point source located at the center of the sea surface, $\boldsymbol{x}=(0,0)$, with a frequency of $f=750 \mathrm{~Hz}$.

A layer of PML elements is placed around the lateral artificial boundaries, see Fig. 7. The domain is discretized using a structured quadrilateral mesh with 10 wavelengths per element, with element size $(\Delta x, \Delta y)=$ $(20,16.7)$. The number of approximation functions after the low-rank reduction is 159 , giving 56,763 DOFs.

Figures 8 and 9 show the different noise patterns produced by an absorbing and a reflecting sea bottom. In the LHS domain region, most part of the acoustic energy penetrates the bottom and the fields are similar 

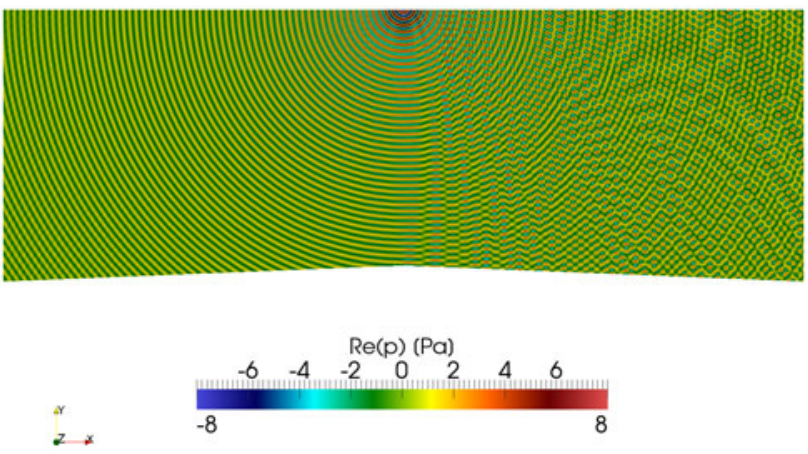

Fig. 8: Detail of the real part of the pressure $(\mathrm{Pa})$ at the central region of the domain $(x \in[-125,+125])$ in Example 2
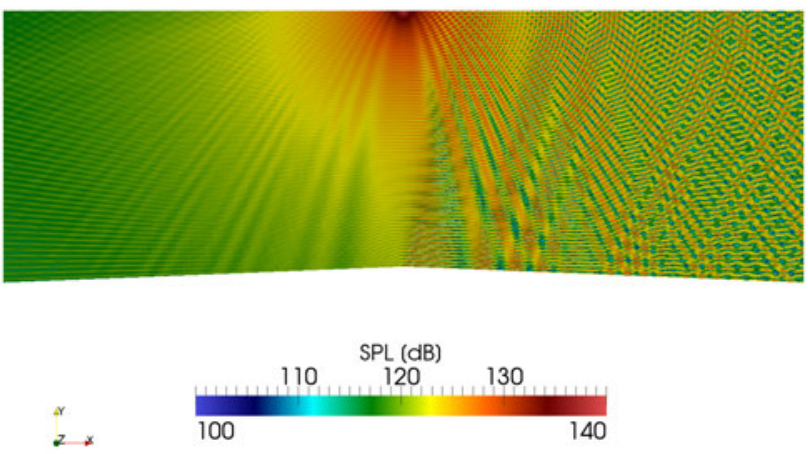

Fig. 9: Detail of the sound pressure level $(\mathrm{dB})$ at the central region of the domain $(x \in[-125,+125])$ in Example 2

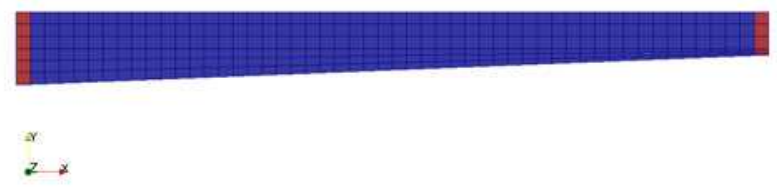

Fig. 10: 2D mesh used in Example 3, including nonPML elements (in blue) and PML elements (in red)

to the ones of the free propagation case. In the RHS region, most energy reflects and the pattern becomes more complex, as a result of the interaction of the incident and reflected wave fronts (the acoustic energy is partially trapped between the surface and the bottom).
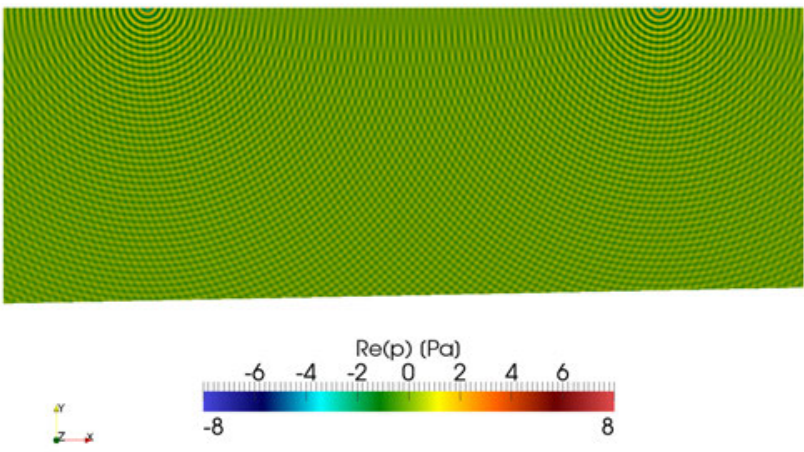

Fig. 11: Detail of the real part of the pressure $(\mathrm{Pa})$ at the central region of the domain $(x \in(-125,+125))$ in Example 3
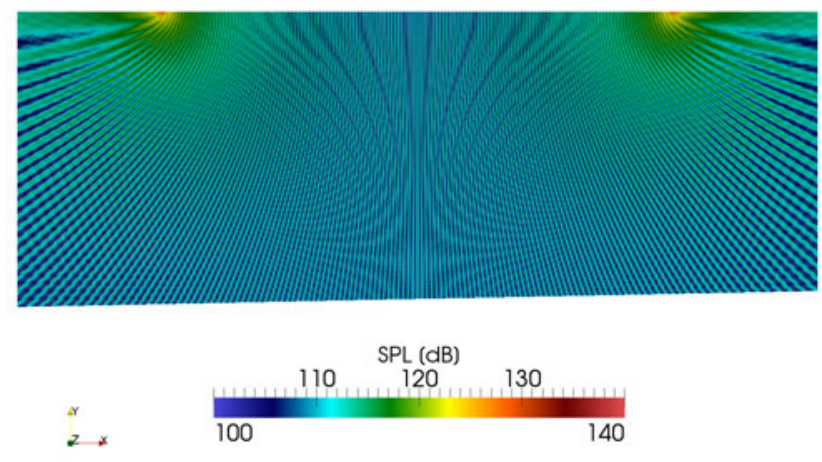

Fig. 12: Detail of the sound pressure level $(\mathrm{dB})$ at the central region of the domain $(x \in(-125,+125))$ in Example 3

4.3 Two sources over a non-horizontal sea bottom with non-uniform sound speed

In this example we consider non-horizontal sea bottom and a non-uniform vertical profile for the sound speed. We set a uniform slope of 0.04 , with a depth varying between $100 \mathrm{~m}$ at $x=-500 \mathrm{~m}$, and $60 \mathrm{~m}$ at $x=500$ $\mathrm{m}$. Two identical sources are placed on the surface at locations $(-80,0)$ and $(+80,0)$. The frequency is $f=$ $750 \mathrm{~Hz}$ and the transmission coefficient at the bottom is $\tau=0.9 i$. The vertical profile of the sound speed is non-uniform: the speed takes a value of $1,500 \mathrm{~m} / \mathrm{s}$ on the surface and decreases linearly with the depth, with a rate of $0.5 \mathrm{~m} / \mathrm{s} / \mathrm{m}(1450 \mathrm{~m} / \mathrm{s}$ at the sea bottom $)$.

The domain is laterally truncated by PMLs, and it is discretized using a structured quadrilateral mesh that includes 10 wavelengths per element, see Fig. 10, with an element size of $(\Delta x, \Delta y)=(20,16.7)$. The number 
of approximation functions per node after the basis reduction is $\tilde{q}=159$, giving 56,763 DOFs.

Figure 11 shows the constructive and destructive interactions between the two pressure fields, while Fig. 12 depicts the resulting sound pressure level map.

\subsection{Sensitivity analysis of the SVD}

This example analyzes the influence of the low-rank approximation of the local enrichment basis on the behavior of the method, paying special attention on the memory footprint, CPU time and accuracy of the proposed method. The key parameter is the tolerance applied to the singular values of the patch matrix, see Eq. (23), and the resulting number of local approximation functions per node, $\tilde{q}$.

We consider the free propagation field generated by a single point source through a half-plane. The domain is truncated creating a rectangular geometry $\Omega=$ $[-500,+500] \times[-100,0]$. The source is located at center of the sea surface, $\boldsymbol{x}=(0,0)$, and generates a noise with a frequency of $f=250 \mathrm{~Hz}$.

The example is solved using 2 meshes with different resolutions. First, we use a mesh with 5 wavelengths per element, giving an element size of $(\Delta x, \Delta y)=(29.4,25)$. Second, we set 10 wavelengths per element, producing an element size of $(\Delta x, \Delta y)=(55.56,50)$. A layer of PML elements is added around the lateral artificial boundaries and below the sea bottom boundary in both cases.

The initial solution space is determined by $q=200$ plane waves per node and, after integrating the standard patch matrix, a SVD is computed, see Sect. 3.4. Then, we select the number of singular values that verify Eq. (23) for $\varepsilon=10^{-16}, 10^{-14}, \ldots, 10^{-2}$. For each tolerance value, we obtain a reduced approximation space with the corresponding number of approximation functions per node, $\tilde{q}$. Since the analytical solution of this problem is provided by Eq. (10), we will assess the accuracy of the solution by computing the relative error in $L^{2}$ norm given by Eq. (25).

Table 1 summarizes, for each tolerance value $\varepsilon$, the most relevant computational parameters and the obtained accuracy, when 5 and 10 wavelengths per element are considered. We highlight that for both meshes, increasing the tolerance $\varepsilon$ (first column) reduces the number of singular values (second column), that is the number of approximation functions per node, leading to a linear reduction in the number of DOFs (third column). This implies an important decrease in the size of the global system matrix or memory footprint (fourth column) and in the CPU time needed to solve the linear system (fifth column), while the relative $L^{2}$-norm error is also reduced (sixth column). It is important to point out that the CPU time involved in the low-rank approximation calculation step is small (below 4 seconds in all cases), compared to the total time of the simulation. In addition, the CPU time for the integration step is almost constant once the number of wavelengths per element is fixed since, in practice, the original approximation functions are always integrated whatever the tolerance value.

As expected, if the original number of shape functions is considered $(q=200)$, the condition number of the generic patch matrix is extremely large, but it decreases as the number of singular values is reduced (seventh column). Moreover, the latter implies a decreasing in the condition number of the global matrix up to 5 orders of magnitude (eighth column).

One of the drawbacks of using enriched solution spaces is that the number of components of the elemental matrices increases with the square of the number of approximation function per node, $q$, leading to large element matrices, and reducing the sparsity of the global matrix. For instance, the ratio of the number of non-zero entries over the total number of entries in this example is $3.6 \%$ when the mesh contains 5 wavelengths per element, and $8.7 \%$ when it contains 10 wavelengths per element. However, in this example we show that a given accuracy can be achieved using a reduced number of combinations of these basis functions. Thus, we can use coarse meshes (large number of wave lengths per element) while keeping the sparsity of the matrix moderate.

Although, for a given accuracy, the use of coarser meshes, with a larger number of wavelengths per elements, requires an increase of the number of enrichment functions per node, numerical experiments show that the increment in the number of approximation functions is compensated by the reduction in the number of mesh nodes, leading to a smaller global number of degrees of freedom, and consequently, to a lower computational cost, see Tab. 1 .

\section{Concluding remarks}

In this paper we have presented a new 2D numerical model to simulate the undersea acoustic propagation generated by multiple non-impulsive (operational) noise sources. The model includes the most relevant physical phenomena such as sea water absorption, via a complex wavenumber, and the reflectance of the sea bottom and surface. Artificial fully absorbing boundary conditions are also included in the lateral boundaries by means of Perfectly Matched Layers. 
Table 1: Required computational resources and achieved accuracy in Example 4, when (a) 5 or (b) 10 wavelengths per element are considered. For each tolerance value we detail: the new number of shape functions $\tilde{q}$ (the initial number is $q=200$ ), the global number of degrees of freedom, the memory space for the global system matrix, the CPU time for the solving step, the relative $L^{2}$-norm error, the condition number of the patch matrix, and the ratio between the condition numbers of the reduced and original system matrices

(a)

\begin{tabular}{cccccccc}
\hline Tol & $\tilde{q}$ & DOF & $K$ storage $(\mathrm{MB})$ & $\mathrm{t}$ solving $(\mathrm{s})$ & $\mathrm{E}$ & $\kappa(K$ patch $)$ & $\kappa(K$ red $) / \kappa(K)$ \\
\hline 0 & 200 & 25,000 & 1597.0 & 2342.85 & 13.8834 & $1.07 \mathrm{E}+16$ & $1.00 \mathrm{E}+00$ \\
$1 \mathrm{E}-16$ & 200 & 25,000 & 1597.0 & 2347.51 & 13.8834 & $1.07 \mathrm{E}+16$ & $1.00 \mathrm{E}+00$ \\
$1 \mathrm{E}-14$ & 128 & 16,000 & 654.2 & 386.21 & 0.1018 & $9.99 \mathrm{E}+13$ & $9.62 \mathrm{E}-01$ \\
$1 \mathrm{E}-12$ & 99 & 12,375 & 391.4 & 172.23 & 0.1028 & $2.43 \mathrm{E}+11$ & $3.58 \mathrm{E}-02$ \\
$1 \mathrm{E}-10$ & 95 & 11,875 & 360.4 & 163.98 & 0.1029 & $5.72 \mathrm{E}+09$ & $2.59 \mathrm{E}-02$ \\
$1 \mathrm{E}-08$ & 89 & 11,125 & 316.3 & 130.77 & 0.1018 & $3.22 \mathrm{E}+07$ & $1.38 \mathrm{E}-02$ \\
$1 \mathrm{E}-06$ & 83 & 10,375 & 275.1 & 105.61 & 0.1017 & $3.39 \mathrm{E}+05$ & $7.34 \mathrm{E}-03$ \\
$1 \mathrm{E}-04$ & 77 & 9,625 & 236.8 & 80.55 & 0.1067 & $7.37 \mathrm{E}+03$ & $3.52 \mathrm{E}-03$ \\
$1 \mathrm{E}-02$ & 67 & 8,375 & 179.3 & 9.09 & 0.1027 & $9.85 \mathrm{E}+01$ & $1.37 \mathrm{E}-04$ \\
\hline
\end{tabular}

(b)

\begin{tabular}{cccccccc}
\hline Tol & $\tilde{q}$ & DOF & $K$ storage $(\mathrm{MB})$ & $\mathrm{t}$ solving $(\mathrm{s})$ & $\mathrm{E}$ & $\kappa(K$ patch $)$ & $\kappa(K$ red $) / \kappa(K)$ \\
\hline 0 & 200 & 11,400 & 558.6 & 183.95 & 0.0759 & $7.78 \mathrm{E}+14$ & $1.00 \mathrm{E}+00$ \\
$1 \mathrm{E}-16$ & 200 & 11,400 & 558.6 & 183.33 & 0.0759 & $7.78 \mathrm{E}+14$ & $1.00 \mathrm{E}+00$ \\
$1 \mathrm{E}-14$ & 193 & 11,001 & 520.2 & 165.17 & 0.0831 & $9.42 \mathrm{E}+13$ & $1.11 \mathrm{E}-03$ \\
$1 \mathrm{E}-12$ & 171 & 9,747 & 408.4 & 120.68 & 0.0716 & $2.82 \mathrm{E}+11$ & $9.78 \mathrm{E}-05$ \\
$1 \mathrm{E}-10$ & 165 & 9,405 & 380.2 & 108.47 & 0.0754 & $2.91 \mathrm{E}+09$ & $7.62 \mathrm{E}-05$ \\
$1 \mathrm{E}-08$ & 159 & 9,063 & 353.1 & 97.66 & 0.0698 & $4.31 \mathrm{E}+07$ & $1.65 \mathrm{E}-04$ \\
$1 \mathrm{E}-06$ & 152 & 8,664 & 322.7 & 87.06 & 0.0768 & $9.75 \mathrm{E}+05$ & $1.29 \mathrm{E}-04$ \\
$1 \mathrm{E}-04$ & 143 & 8,151 & 285.6 & 70.67 & 0.0794 & $6.43 \mathrm{E}+03$ & $4.34 \mathrm{E}-04$ \\
$1 \mathrm{E}-02$ & 127 & 7,239 & 225.3 & 50.99 & 0.1075 & $8.44 \mathrm{E}+01$ & $3.13 \mathrm{E}-04$ \\
\hline
\end{tabular}

We have successfully used the Partition of the Unity Method to solve the corresponding weak form of the Helmholtz equation, since harmonic noise sources are heeded. Specifically, we enrich the solution space by pasting several plane waves with the physical wavenumber at each patch of the discretization. Two basic ingredients are proposed to properly solve the weak form. On the one hand we have implemented a semi-analytical scheme in order to integrate highly oscillatory functions over quadrilateral elements. This allows reducing the computational cost of the integration step while maintaining its accuracy. On the other hand, we have developed a new procedure to reduce the condition number of the elemental and global matrices. It is based on a low-rank approximation of the local enrichment basis associated to the central node of a reference patch. This way, we have reduced the size of the global matrix while preserving the accuracy of the approximation.

We have applied the model to several scenarios including absorbing medium, sea bottom composed of different materials, and non-planar sea bottom. In addition, we have computed the free propagation of the sound generated by a single point source through a lossless and through a lossy media, and compared it with the analytical solution. In both cases we obtained accurate results. Finally, we have analyzed the influence of the low-rank approximation on the behavior of the proposed method. Numerical experiments show that by reducing the number of local approximation functions, we can also reduce the required computational resources and the accuracy of the results.

Several aspects of the proposed model have to be further investigated and improved in the near future. We can improve our strategy for obtaining the low-rank approximations of the local basis. The current approach is based on a patch around a given node, without taking into account the interaction between the approximation functions associated to different nodes. We claim that the reduction in the condition number of the global system matrix can be improved by developing a criterion based on a low-rank approximation space at the element level. Thus, a better conditioning will be obtained with a lower computational cost (both in terms of CPU time and memory footprint) for the same accuracy.

In our model we introduce the input noise integrating the independent term of the Robin boundary condition along the boundary edges of a set of elements adjacent to the noise source. This increases the error 
of the solution in regions located near the sea surface and far of the input noise sources. We suggest that this can be overcome by introducing the input noise via the independent term of the Helmholtz equation, using a Dirac delta function.

Iterative solvers have to be considered in order to increase the computational efficiency of the simulation tool. Even though the global number of degrees of freedom is moderate, the fact that each node includes multiple degrees of freedom leads to the assembly of large elemental matrices that dramatically reduce the sparsity of the system matrix, compared to the typical sparsity associated to standard Finite Element approaches. Thus, the fill-in generated by direct solvers leads to large memory requirements, and the CPU time involved in the solver step may be prohibitive (specially if the full set of initial plane wave directions is considered).

The accuracy of the method can be improved using an adaptive strategy. Therefore, an a-posteriori error estimation framework has to be developed. From these error estimates an adaptivity process could be deduced to determine the optimal number of planes waves that has to be pasted at each node (q-adaptivity).

Finally, the proposed model can be extended to 3D simulations. To this end, first we need to find an initial uniformly distributed set of three-dimensional plane wave directions and a semi-analytical integration rule for 3D elements. Moreover, the development of specific iterative solvers that take into account the structure of the system matrices, and the implementation of improved low-rank approximations will play a major role to reduce the computational resources involved in this kind of simulations.

\section{A Seawater absorption}

In this appendix we deduce the relation between the absorption coefficient $\alpha$ and the imaginary part of the complex wavenumber $k_{2}$, see Eq. (7). The value of the seawater absorption coefficient $\alpha$ strongly depends on the frequency, but also on the temperature, the salinity, the hydrostatic pressure (depth) and the acidity. Our model estimates the coefficient by means of the Ainslie and McColm formula [40]:

$$
\begin{aligned}
\alpha & =0.106 \exp ((\mathrm{pH}-8) / 0.56) \frac{f_{1} f^{2}}{f_{1}^{2}+f^{2}} \\
& +0.52\left(1+\frac{T}{43}\right)\left(\frac{S}{35}\right) \exp (-d / 6) \frac{f_{2} f^{2}}{f_{2}^{2}+f^{2}} \\
& +0.00049 \exp (-(T / 27+d / 17)) f^{2},
\end{aligned}
$$

where

$f_{1}=0.78\left(\frac{S}{35}\right)^{1 / 2} \exp (T / 26)$

is the boron acid relaxation frequency in $\mathrm{kHz}$,

$f_{2}=42 \exp (T / 17)$

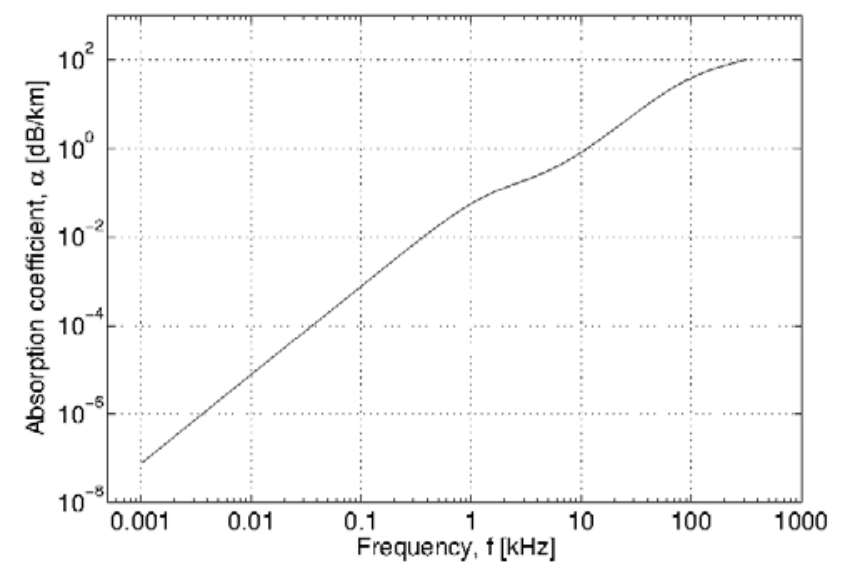

Fig. 13: Absorption coefficient $\alpha$ under the following conditions: $S=35 \mathrm{ppt}, T=17^{\circ}, d=0 \mathrm{~km}, \mathrm{pH}=8$

is the magnesium sulfate relaxation frequency in $\mathrm{kHz}, \alpha$ is the absorption coefficient in $\mathrm{dB} / \mathrm{km}, f$ is the frequency in $\mathrm{kHz}$, $T$ is the seawater temperature in ${ }^{\circ} \mathrm{C}, S$ is the salinity in ppt, $d$ is the depth in $\mathrm{km}$, and $\mathrm{pH}$ is the measure of the acidity.

If part of data is missing, Eq. (26) can be replaced by

$\alpha=0.159 \frac{f^{2}}{2.25+f^{2}}+30.5 \frac{f^{2}}{1764+f^{2}}+0.000261 f^{2}$,

that considers the average conditions at the ocean surface: $T$ $=17^{\circ} \mathrm{C}, S=35 \mathrm{ppt}$, and $d=0 \mathrm{~km}$ and $\mathrm{pH}=8$. Figure 13 shows the dependence of the absorption coefficient with the frequency according to Eq. (27). Note that the absorption is specially significant for frequencies above a few $\mathrm{kHz}$, and that can be neglected for short and mid-range propagations at low frequency.

Once the absorption coefficient $\alpha$ is computed using Eq. (26) or Eq (27), it is included in our model via the the imaginary part of the complex wavenumber $k_{2}$. Given a plane wave with an arbitrary amplitude $A$, propagating in a free space following an arbitrary direction vector $e$, it suffers an exponential decay determined by $k_{2}$,

$W(\boldsymbol{x})=A \exp (i k \boldsymbol{e} \cdot \boldsymbol{x})=A \exp \left(-k_{2} \boldsymbol{e} \cdot \boldsymbol{x}\right) \exp \left(i k_{1} \boldsymbol{e} \cdot \boldsymbol{x}\right)$.

Thus, and according to Eqs. (1) and Eq. (28), the transition loss between two points separated by a distance $r$ (in $\mathrm{km}$ ) produced exclusively by the physical absorption is:

$$
\begin{aligned}
\alpha r & =\mathrm{SPL}_{0}-\mathrm{SPL}_{r}=20 \log _{10} \frac{p_{\mathrm{rms}, 0}}{p_{\text {ref }}}-20 \log _{10} \frac{p_{\mathrm{rms}, r}}{p_{\text {ref }}} \\
& =20 \log _{10}\left[\exp \left(k_{2} \cdot 1000 r\right)\right],
\end{aligned}
$$

and Eq. (7) follows.

\section{B Transmission coefficient}

In this appendix we relate the transmission coefficient $\tau$ appearing in the Robin boundary condition (3) with several material properties of the surrounding media (air and sea bottom). The transmission coefficient can be written as [45]:

$\tau=\tau_{1}+i \tau_{2}$, 
where $i=\sqrt{-1}$ is the imaginary unit,

$\tau_{1}=\frac{2 K_{r} \sin \beta \cos \gamma}{1+K_{r}^{2}+2 K_{r} \cos \beta}$

and

$\tau_{2}=\frac{\left(1-K_{r}^{2}\right) \cos \gamma}{1+K_{r}^{2}+2 K_{r} \cos \beta}$,

being $K_{r}$ the reflection coefficient, which is the ratio between the reflected and the incident wave amplitudes $K_{r}=$ $\left|p_{r}\right| /\left|p_{i}\right|, \beta$ the reflection phase angle, and $\gamma$ the incident wave direction relative to the normal at the boundary.

The reflection phase angle $\beta$ is set to zero, considering that the position of the numerical boundary agrees with its actual position. Thus, the complex transmission coefficient is purely imaginary. In addition, the incident wave direction $\gamma$ cannot be unambiguously specified since the full-wave approach implies multiple reflections with different wave incident directions angles. In our model we conservatively assume normal incidence $(\gamma=0)$. This is conservative in the sense that the produced noise level is going to be larger than the actual one. Thus,

$\tau=i \frac{1-K_{r}}{1+K_{r}}$.

The value of the transmission coefficient $K_{r}$ can be obtained either from the value of the transmission loss at the interface $\mathrm{TL}_{i}$, measured in $\mathrm{dB}$, or from the acoustic impedance $Z_{b}=\rho_{b} c_{b}$ of the boundary, being $\rho_{b}$ the density of the boundary material, depending on the availability of empirical measurements. In the first case, we have

$K_{r}=10^{-\mathrm{TL}_{i} / 20}$.

In the second case, assuming normal incidence, we have

$K_{r}=\frac{Z_{b} / Z_{s w}-1}{Z_{b} / Z_{s w}+1}$,

where $Z_{s w} \approx 1.54 \times 10^{6} \mathrm{~kg} /\left(\mathrm{m}^{2} \mathrm{~s}\right)$ is the acoustic impedance of the seawater. Substituting Eq. (30) and Eq. (31) in Eq. (29) we obtain expression (8) and (9), respectively.

\section{References}

1. J. R. Nedwell, J. Langworthy, and D. Howell, "Assessment of sub-sea acoustic noise and vibration from offshore wind turbines and its impact on marine wildlife; initial measurements of underwater noise during construction of offshore windfarms, and comparison with background noise," Subacoustech Report ref: 544R0424, published by COWRIE, May 2003.

2. M. A. Ainslie, C. A. F. de Jong, H. S. Dol, G. Blaquière, and C. Marasini, "Assessment of natural and anthropogenic sound sources and acoustic propagation in the North Sea," TNO report TNO-DV, p. C085, Feb. 2009.

3. F. Thomsen, K. Lüdemann, R. Kafemann, and W. Piper, "Effects of offshore wind farm noise on marine mammals and fish," Biola, Hamburg, Germany on behalf of COWRIE Ltd, p. 62, July 2006.

4. F. Thomsen, "Assessment of the environmental impact of underwater noise," OSPAR Commission. Biodiversity Series, vol. 436, 2009.
5. B. L. Southall, A. E. Bowles, W. T. Ellison, J. J. Finneran, R. L. Gentry, C. L. Greene Jr., D. Kastak, D. R. Ketten, J. H. Miller, P. E. Nachtigall, W. J. Richardson, J. A. Thomas, and P. L. Tyack, "Marine mammal noise exposure criteria: initial scientific recommendations," Aquatic Mammals, vol. 33, no. 4, pp. 411$521,2007$.

6. Marine Mammal Commission, "The Marine Mammal Protection Act of 1972," 1972.

7. O. Commission et al., "Convention for the Protection of the Marine Environment of the North-East Atlantic," 1992.

8. "Directive 2008/56/EC establishing a framework for community action in the field of marine environmental policy (Marine Strategy Framework Directive)," June 2008.

9. R. P. A. Dekeling, M. L. Tasker, M. A. Ainslie, M. Andersson, M. Andr, M. Castellote, J. F. Borsani, J. Dalen, T. Folegot, R. Leaper, A. Liebschner, J. Pajala, S. P. Robinson, P. Sigray, G. Sutton, F. Thomsen, A. J. Van der Graaf, S. Werner, D. Wittekind, and J. V. Young, "Monitoring Guidance for Underwater Noise in European Seas," Second Report of the Technical Subgroup on Underwater Noise (TSG Noise), May 2013.

10. R. A. Hazelwood and J. Connelly, "Estimation of underwater noise - a simplified method," Int J Soc for Underw Technol, vol. 26, no. 3, pp. 51-57, 2005.

11. F. D. Tappert, The parabolic approximation method, vol. 70 of Lecture Notes in Physics, ch. 5, pp. 224-287. Courant Institute of Mathematical Sciences, New York University: Springer, 1977.

12. M. D. Collins, "A split-step Padé solution for the parabolic equation method," J Acoust Soc Am, vol. 93, pp. 1736-1742, Apr. 1993

13. I. Babuška and S. A. Sauter, "Is the pollution effect of the FEM avoidable for the Helmholtz equation considering high wave numbers?," SIAM J Numer Anal, vol. 34, pp. 2392-2423, 1997.

14. F. Ihlenburg, Finite Element Analysis of Acoustic Scattering, vol. 132 of Applied Mathematical Sciences. New York: Springer-Verlag, 1st ed., 1998.

15. A. Deraemaeker, I. Babuška, and P. Bouillard, "Dispersion and pollution of the FEM solution for the Helmholtz equation in one, two and three dimensions," Int J Numer Methods Engrg, vol. 46, pp. 471-499, 1999.

16. J. M. Melenk, On Generalized Finite Element Methods. $\mathrm{PhD}$ thesis, The University of Maryland, 1995.

17. J. M. Melenk and I. Babuška, "The partition of unity finite element method: Basic theory and applications," Comput Methods Appl Mech Engrg, vol. 139, pp. 289314, 1996.

18. I. Babuška and J. M. Melenk, "The partition of unity method," Int J Numer Methods Engrg, vol. 40, pp. 727758, 1997.

19. I. Herrera and F. J. Sabina, "Connectivity as an alternative to boundary integral equations: Construction of bases," Proc Natl Acad Sci USA, vol. 75, pp. 2059-2063, May 1978.

20. E. Perrey-Debain, O. Laghrouche, P. Bettess, and J. Trevelyan, "Plane-wave basis finite elements and boundary elements for three-dimensional wave scattering," Phil. Trans. R. Soc. Lond. A, vol. 362, pp. 561-577, Mar. 2004.

21. D. Wang, R. Tezaur, J. Toivanen, and C. Farhat, "Overview of the Discontinuous Enrichment Method, the Ultra-Weak Variational Formulation, and the Partition of Unity Method for acoustic scattering in the medium 
frequency regime and performance comparisons," Int $J$ Numer Methods Engrg, vol. 89, pp. 403-417, 2012.

22. O. Cessenat and B. Després, "Application of an ultra weak variational formulation of elliptic PDEs to the twodimensional Helmholtz problem," SIAM J Numer Anal, vol. 35, pp. 255-299, Feb. 1998.

23. T. Huttunen, P. Monk, and J. P. Kaipio, "Computational Aspects of the Ultra-Weak Variational Formulation," $J$ Comput Phys, vol. 182, pp. 27-46, July 2002.

24. C. Farhat, I. Harari, and L. P. Franca, "The Discontinuous Enrichment Method," Comput Methods Appl Mech Engrg, vol. 190, pp. 6455-6479, 2001.

25. C. Farhat, I. Harari, and U. Hetmaniuk, "A discontinuous Galerkin method with Lagrange multipliers for the solution of Helmholtz problems in the mid-frequency regime," Comput Methods Appl Mech Engrg, vol. 132, pp. 13891419, 2003.

26. I. Babuška, F. Ihlenburg, E. T. Paik, and S. A. Sauter, "A Generalized Finite Element Method for solving the Helmholtz equation in two dimensions with minimal pollution," Comput Methods Appl Mech Engrg, vol. 128, pp. 325-359, 1995.

27. P. Mayer and J. Mandel, "The finite ray element method for the Helmholtz equation of scattering: first numerical experiments," Oct. 1997.

28. O. Laghrouche and P. Bettess, "Short wave modelling using special finite elements," J Comput Acoust, vol. 8, no. 1, pp. 189-210, 2000.

29. P. Ortiz and E. Sanchez, "An improved partition of unity finite element model for diffraction problems," Int $J \mathrm{Nu}$ mer Methods Engrg, vol. 50, pp. 2727-2740, 2001.

30. O. Laghrouche, P. Bettess, and R. J. Astley, "Modelling of short wave diffraction problems using approximating systems of plane waves," Int J Numer Methods Engrg, vol. 54, pp. 1501-1533, 2002.

31. O. Laghrouche, P. Bettess, E. Perrey-Debain, and J. Trevelyan, "Plane wave basis finite-elements for wave scattering in three dimensions," Commun Numer Meth Engrg, vol. 19, pp. 715-723, 2003.

32. P. Ortiz, "Finite elements using a plane-wave basis for scatering of surface water waves," Phil Trans $R$ Soc London A, vol. 362, pp. 525-540, 2004.

33. O. Laghrouche, P. Bettess, E. Perrey-Debain, and J. Trevelyan, "Wave interpolation finite elements for Helmholtz problems with jumps in the wave speed," Comput Methods Appl Mech Engrg, vol. 194, pp. 367381, 2005.

34. E. De Bel, P. Villon, and P. Bouillard, "Forced vibrations in the medium frequency range solved by a partition of unity method with local information," Int J Numer Methods Engrg, vol. 62, pp. 1105-1126, Nov. 2005.

35. T. Strouboulis and R. Hidajat, "Partition of unity method for Helmholtz equation: $q$-convergence for plane waves and wave-band local bases," Appl Math, vol. 51, no. 2, pp. 181-204, 2006.

36. P. Bettess, J. Shirron, O. Laghrouche, B. Peseux, R. Sugimoto, and J. Trevelyan, "A numerical integration scheme for special finite elements for the Helmholtz equation," Int J Numer Methods Engrg, vol. 56, pp. 531-552, 2003.

37. R. Sugimoto, P. Bettess, and J. Trevelyan, "A numerical integration scheme for special quadrilateral finite elements for the Helmholtz equation," Commun Numer Meth Engrg, vol. 19, pp. 233-245, 2003.

38. W. A. Kuperman and J. F. Lynch, "Shallow-Water Acoustics," Physics Today, vol. 57, pp. 55-61, Oct. 2004.

39. European Wind Energy Association (EWEA)and others, "Deep Water - The next step for offshore wind energy,"
Brussels, Belgium: A report by the European Wind Energy Association, July 2013.

40. M. A. Ainslie and J. G. McColm, "A simplified formula for viscous and chemical absorption in sea water," $J$ Acoust Soc Am, vol. 103, pp. 1671-1672, Mar. 1998.

41. G. S. K. Wong and S. Zhu, "Speed of sound in seawater as a function of salinity, temperature, and pressure," $J$ Acoust Soc Am, vol. 97, pp. 1732-1736, Mar. 1995.

42. C.-T. Chen and F. J. Millero, "Speed of sound in seawater at high pressures," J Acoust Soc Am, vol. 62, pp. 11291135, Nov. 1977.

43. C. C. Leroy and F. Parthiot, "Depth-pressure relationships in the oceans and seas," J Acoust Soc Am, vol. 103, pp. 1346-1352, Mar. 1998.

44. M. Isaacson and $\mathrm{S}$. $\mathrm{Qu}$, "Waves in a harbour with partially reflecting boundaries," Coast Engrg, vol. 14, pp. 193-214, 1990.

45. J. C. W. Berkhoff, Mathematical models for simple harmonic linear water waves. Wave diffraction and refraction. PhD thesis, TU Delft, Delft, Nederlands, Apr. 1976.

46. R. J. Urick, Principles of Underwater Sound. Los Altos, California: Peninsula Publishing, third ed., 1983.

47. W. S. Burdic, Underwater Acoustic System Analysis. Prentice-Hall signal processing series, London: PrenticeHall, Inc., 1983.

48. C. M. Rappaport, "Perfectly matched absorbing boundary conditions based on anisotropic lossy mapping of space," IEEE Microw Guided Wave Letters, vol. 5, pp. 90-92, Mar. 1995.

49. J.-P. Bérenger, "A Perfectly Matched Layer for the Absorption of Electromagnetic Waves," J Comput Phys, vol. 114, pp. 185-200, 1994.

50. S. G. Johnson, "Notes on Perfectly Matched Layers (PMLs)." Notes for MIT courses 18.369 and 18.336, Aug. 2007.

51. L. E. Kinsler, A. R. Frey, A. B. Coppens, and J. V. Sanders, Fundamentals of Acoustics. New York, USA: John Wiley \& Sons, fourth ed., 2000.

52. T. Strouboulis, I. Babuška, and R. Hidajat, "The generalized finite element method for Helmholtz equation: Theory, computation, and open problems," Comput Methods Appl Mech Engrg, vol. 195, pp. 4711-4731, 2006.

53. T. Strouboulis, R. Hidajat, and I. Babuška, "The generalized finite element method for Helmholtz equation. Part II: Effect of choice of handbook functions, error due to absorbing boundary conditions and its assessment," Comput Methods Appl Mech Engrg, vol. 197, pp. 364$380,2008$. 